\title{
Quasistatic delamination of sandwich-like Kirchhoff-Love plates
}

\author{
Lorenzo Freddi ${ }^{1}$, Tomáš Roubíčé ${ }^{2,3}$, Chiara Zanini ${ }^{4}$
}

\footnotetext{
${ }^{1}$ Dipartimento di Matematica e Informatica, Università di Udine, Via delle Scienze 206, I-33100 Udine, Italy.

2 Mathematical Institute, Charles University, Sokolovská 83, CZ-186 75 Praha 8, Czech Republic,

${ }^{3}$ Institute of Thermomechanics of the ASCR, Dolejškova 5, CZ-182 00 Praha 8, Czech Republic.

${ }^{4}$ Dipartimento di Scienze Matematiche, Politecnico di Torino, Corso Duca degli Abruzzi 24, 10129 Torino, Italy.
}

Accepted paper (2012)

Journal of Elasticity, DOI: 10.1007/s10659-012-9419-9

\begin{abstract}
A quasistatic rate-independent adhesive delamination problem of laminated plates with a finite thickness is considered. By letting the thickness of the plates go to zero, a rate-independent delamination model for a laminated Kirchhoff-Love plate is obtained as limit of these quasistatic processes. The same dimension reduction procedure is eventually applied to processes which are sensitive to delamination modes, namely opening vs. shearing is distinguished.
\end{abstract}

Key Words. Adhesive contact, delamination, mixed modes, rate-independent processes, dimension reduction, $\Gamma$-convergence, Kirchhoff-Love plates.

AMS Subject Classification: 49J45, 49S05, 74K20, 74R10.

\section{Introduction, notation, basic concepts}

This paper aims to study delamination of sandwich-like laminated plates. In particular, a two-dimensional model for the quasistatic evolution of the delamination process is rigorously derived by dimension reduction, starting from a three-dimensional theory of adhesive contact between two plates.

We base our analysis on $\Gamma$-convergence. The $\Gamma$-convergence method to study dimension reduction problems has been introduced, in the static case, by Acerbi, Buttazzo and Percivale [1], for nonlinear elastic strings. This approach has been followed soon by Anzellotti, Baldo, Percivale [3] and Borquin, Ciarlet, Geymonat, Raoult [7] for linear elastic beams and plates. It also led to the first convergence result for nonlinear elastic plates due to Le Dret and Raoult [39] who themselves based their approach on that of [1]. $\Gamma$-convergence provides an alternative to the more classical formal asymptotic development methods that, since Ciarlet and Destuynder [12], are based on a variational weak formulation of the problem. The notion of quasi-static evolution in fracture mechanics, as addressed by Francfort and Marigo [17,18], is based on an energy minimization procedure which fits the energetic solution framework of Mielke and Theil [51], for which it is natural to apply $\Gamma$-convergence methods.

We confine ourselves to small strains. The surface where delamination may occur is assumed to be apriori prescribed. Furthermore, we restrict our attention to quasistatic unidirectional (i.e., healing of the adhesive is not possible) rate-independent delamination. We consider a unilateral Signorini contact, which is important to prevent (unphysical) delamination by mere compression. In the first part of the paper, we focus on a standard, mode-insensitive delamination, while, in Section 5 we distinguish between various modes of delamination (namely opening vs. shearing). The variational dimension reduction process leads to a Kirchhoff-Love model for the plate. The case of a "vertically" positioned delaminating surface has been studied in [21] and, using a Barenblatt-like cohesive crack surface energy, also in [20]. For a static delamination on a generally-positioned delamination surface in Kirchhoff-Love plates, we refer to $[29,30]$. 

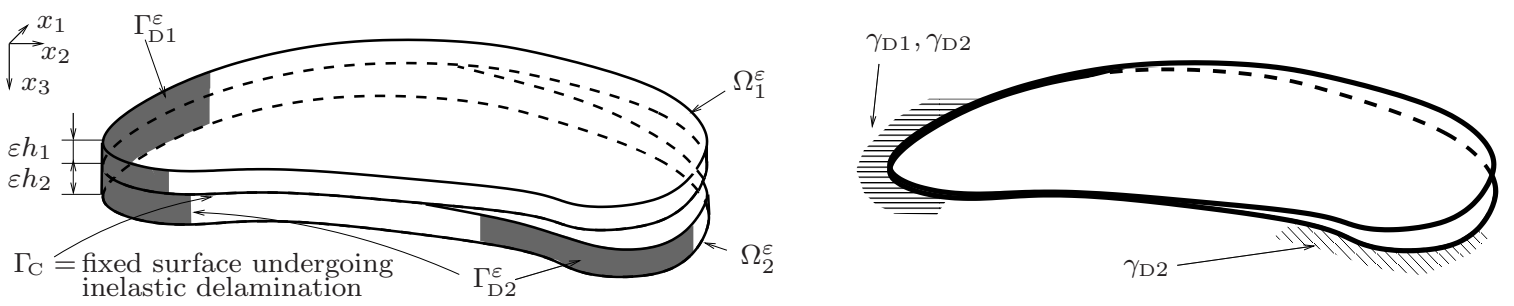

Fig. 1. Illustration of the geometry and of the notation.

Left: a $3 D$ thin plate-like body undergoing delamination on a prescribed surface $\Gamma_{\mathrm{C}}$.

Right: $2 D$ plates obtained for $\varepsilon \rightarrow 0$ undergoing delamination.

For notational simplicity, we study the case of only two plates glued together by one delamination surface $\Gamma_{\mathrm{C}}$, dividing thus a 3-dimensional cylindrical elastic body into two parts occupying respectively the domains $\Omega_{1}^{\varepsilon}$ and $\Omega_{2}^{\varepsilon}$, cf. Figure 1(left). The generalization of a sandwich laminate composed from $N \geq 3$ domains glued on $N-1$ surfaces is straightforward.

We assume both parts $\Omega_{1}^{\varepsilon}$ and $\Omega_{2}^{\varepsilon}$ to be fixed by Dirichlet boundary conditions on some (non-vanishing) parts of the side boundary, denoted by $\Gamma_{\mathrm{D} 1}^{\varepsilon}$ and $\Gamma_{\mathrm{D} 2}^{\varepsilon}$, respectively; cf. again Figure 1(left). We consider a rather special "cylindrical" case, i.e., in particular the Dirichlet parts of the boundary are positioned vertically, while the delaminating surface $\Gamma_{\mathrm{C}}$ is horizontal. More precisely,

$$
\Omega_{1}^{\varepsilon}:=\Gamma_{\mathrm{C}} \times\left(-\frac{\varepsilon h_{1}}{2}, 0\right), \quad \Omega_{2}^{\varepsilon}:=\Gamma_{\mathrm{C}} \times\left(0, \frac{\varepsilon h_{2}}{2}\right), \quad \Gamma_{\mathrm{D} 1}^{\varepsilon}=\gamma_{\mathrm{D} 1} \times\left(-\frac{\varepsilon h_{1}}{2}, 0\right), \quad \Gamma_{\mathrm{D} 2}^{\varepsilon}=\gamma_{\mathrm{D} 2} \times\left(0, \frac{\varepsilon h_{2}}{2}\right) .
$$

We will use the abbreviation

$$
\Omega^{\varepsilon}=\Omega_{1}^{\varepsilon} \cup\left(\Gamma_{\mathrm{C}} \times\{0\}\right) \cup \Omega_{2}^{\varepsilon}=\Gamma_{\mathrm{C}} \times\left(-\frac{\varepsilon h_{1}}{2}, \frac{\varepsilon h_{2}}{2}\right) \quad \text { and } \quad \Gamma_{\mathrm{D}}^{\varepsilon}=\Gamma_{\mathrm{D} 1}^{\varepsilon} \cup \Gamma_{\mathrm{D} 2}^{\varepsilon} .
$$

The constant parameters $h_{1}$ and $h_{2}$ are kept to clarify the role played by the thickness of the body in the limit problem. This will allow for the dimensional reduction by letting the aspect ratio $\varepsilon$ go to 0 . The geometry of the resulted laminated-composite plate (here, for simplicity, composed only of two plates) is then depicted in Figure 1(right).

Thoroughout the whole article, we will use a rather special general framework, namely that the state, denoted by $q=(u, z)$, and its evolution are governed by a time-dependent stored energy, denoted by $\mathcal{E}$, and a potential of dissipative forces, denoted by $\mathcal{R}$. This potential depends only on the rate $\dot{z}$ of the dissipative component $z$ but not on the state $q$ itself (the so-called associative case) and is positively homogeneous (the so-called rate-independent case). The quasistatic evolution we have in mind is governed by the following Biot's type [5,6] system of doubly nonlinear degenerate parabolic/elliptic variational inclusions:

$$
\partial_{u} \mathcal{E}(t, u, z) \ni 0 \text { and } \partial \mathcal{R}(\dot{z})+\partial_{z} \mathcal{E}(t, u, z) \ni 0,
$$

where " $\partial$ " denotes the usual (partial) subdifferential of convex analysis, according with the fact that, throughout the paper, the functionals $\mathcal{R}(\cdot), \mathcal{E}(t, \cdot, z)$, and $\mathcal{E}(t, u, \cdot)$ are convex.

An important assumption is that $\mathcal{R}$ is degree- 1 positively homogeneous, which implies (and even is equivalent to) that the dissipation rate is just the potential of a dissipative force. Also this implies that, if $\mathcal{E}(t, \cdot, \cdot)$ is convex, the conventional weak solutions are basically equivalent (under mild additional temporal regularity assumptions) to so-called energetic solutions of the rate-independent system associated to $\mathcal{E}$ and $\mathcal{R}$ with the initial conditions

$$
u(0)=u^{0} \quad \text { and } \quad z(0)=z^{0} .
$$

We consider a fixed time horizon $T>0$ and denote by $\mathcal{U}$ and $\mathcal{z}$ the abstract spaces where $u$ and $z$ live, respectively.

Definition 1.1 (Energetic solution). The process $q=(u, z):[0, T] \rightarrow \mathcal{Q}:=\mathcal{U} \times \mathcal{Z}$ is called an energetic solution of the initial-value problem given by $\left(\mathcal{U} \times \mathcal{Z}, \mathcal{E}, \mathcal{R}, u^{0}, z^{0}\right)$ if, beside $(1.3), t \mapsto \partial_{t} \mathcal{E}(t, q(t)) \in L^{1}((0, T))$, 
if for all $t \in[0, T]$ we have $\mathcal{E}(t, q(t))<+\infty$ and if the global stability inequality (1.4a) and the global energy balance (1.4b) are satisfied for all $t \in[0, T]$ :

$$
\begin{aligned}
& \forall \check{q}=(\check{u}, \check{z}) \in \mathcal{Q}: \quad \mathcal{E}(t, q(t)) \leq \mathcal{E}(t, \check{q})+\mathcal{R}(\check{z}-z(t)), \\
& \mathcal{E}(t, q(t))+\operatorname{Diss}_{\mathcal{R}}(z,[0, t])=\mathcal{E}(0, q(0))+\int_{0}^{t} \partial_{s} \mathcal{E}(s, q(s)) \mathrm{d} s
\end{aligned}
$$

with $\operatorname{Diss}_{\mathcal{R}}(z,[0, t]):=\sup \sum_{j=1}^{N} \mathcal{R}\left(z\left(t_{j}\right)-z\left(t_{j-1}\right)\right)$, where the supremum is taken over all partitions $0 \leq$ $t_{0}<t_{1}<\ldots<t_{N} \leq t$ of $[0, t]$ with $N \in \mathbb{N}$.

If $\mathcal{E}(t, \cdot, \cdot)$ is separately convex but nonconvex, as in this work, then (1.2) and (1.4) are no longer equivalent. The energetic formulation (1.4) then represents a generalized formulation based on a minimumenergy principle competing with the maximum-dissipation principle or rather with Levitas' realizability principle [41], cf. $[46,51,52]$. The main advantages of the energetic-solution concept are that it is derivative-free, i.e., there is no $\partial_{u} \mathcal{E}$, nor $\partial_{z} \mathcal{E}$, neither $\dot{z}$ in Definition 1.1, and that it can be handled by Calculus of Variations techniques (in particular variational convergence, as shown in [50] and as exploited also here) as well as strictly linked with direct numerical methods, as shown in [49]. For its application to delamination, namely to the problem determined below by $\left(\mathcal{U} \times \mathcal{Z}, \mathrm{E}_{\varepsilon}, \mathrm{R}_{\varepsilon}^{d}, \mathrm{q}_{\varepsilon}^{0}\right)$, with numerical implementation and computational simulations we refer to $[35,56,59]$. Roughly speaking, energetic solutions tempt to evolve as soon as it is energetically convenient. This may, however, not be exactly always in full agreement with the response of real systems involving some other rate-dependent phenomena. Therefore, in spite of these theoretical and computational arguments supporting the energetic-solution concept, there are also some other concepts of solutions that are sometimes applicable and successfully competing with energetic solutions, cf. also $[16,47,48,53]$ for a comparison with other notions in general, and, e.g., $[10,31-33,36,37,54,55]$ in the context of crack propagation, or, e.g., $[4,13,14]$ in the context of plasticity. Moreover, we refer to e.g. $[8,9,27,28,40]$ for some more classical approaches used in the engineering literature. The validity of the dimension reduction results presented here for the energeticsolution concept is not obvious in the case of other solution concepts.

One should emphasize the quite essential differences from a delamination surface being positioned "vertically", which was investigated in $[20,21]$. In those papers the vertically positioned surface scaled as $\varepsilon$, in contrast to the horizontally positioned $\Gamma_{\mathrm{C}}$ considered in this paper. Due to this fact, the energies have here a different scaling, cf. (3.6) and (3.10) below. Another difference due to the "sandwich" position of the contact surface is that the delamination parameter $z$ is now independent of $x_{3}$, which means that here it will not be rescaled and remains the same also for the limit 2D problem. Moreover, [21] considers also a delamination model obtained for $\kappa_{\tau}=\kappa_{\nu}=\kappa \rightarrow+\infty$, cf. (3.6). This leads to a so-called brittle, Griffith-type delamination model, but such a limit passage is not clear in the framework of the present work.

It is worth notice that a related interesting issue concernes the study of the singularities developed by the energetic solutions, see for instance $[2,15,25,34,58]$, including their propagation in the quasi-static evolution process. Nevertheless this kind of analysis relies on completely different techniques and for this reason it is out of the purposes of the present paper.

The paper is organized as follows: In Sect. 2 we formulate the original 3-dimensional problem. In Sect. 3 we specify the scaling we want to consider, rescale the original problem to a fixed 3-dimensional domain, and state the convergence result. In Sect. 4 we formulate a 2-dimensional variant of the limit problem. Eventually, Sect. 5 investigates a generalization allowing to distinguish among different delamination modes, namely opening from shearing or from mixing of both.

We will use the standard notation as far as the function spaces concerns: $C^{k}$ for the space of functions with continuous $k$-th derivative, $L^{p}$ for Lebesgue spaces and $W^{k, p}$ for Sobolev spaces. Given a vector function $v$, the $\alpha$-th component of $v$, usually denoted by $v_{\alpha}$, will be sometimes denoted also with $[v]_{\alpha}$.

Considering the continuous parameter $\varepsilon \rightarrow 0+$, cf. Fig. 1(left), for notational simplicity and without any confusion, when speaking about a sequence we have in mind a countable number of $\varepsilon$ 's, $\varepsilon_{n}$, converging to 0 . So, when we write $\mathcal{E}_{\varepsilon}$ in fact we have in mind $\mathcal{E}_{\varepsilon_{n}}$, and so on. We immediately make use of this notation in introducing the concept of uniformly stable sequence which plays a basic role in stating and proving our convergence results and in particular in the construction of a so-called mutual recovery sequence, cf. (3.21b) below. 
As in Definition 1.1, we consider $\mathcal{Q}=\mathcal{U} \times \mathcal{Z}$ and set $q=(u, z)$, and we shall write, for instance, $(t, q)$ in place of $(t, u, z)$. For $t \in[0, T]$ and $\varepsilon \geq 0$, the sets of stable states $\mathcal{S}_{\varepsilon}(t)$ is defined as

$$
\mathcal{S}_{\varepsilon}(t):=\left\{q \in \mathcal{Q}: \mathcal{E}_{\varepsilon}(t, q)<+\infty \text { and } \mathcal{E}_{\varepsilon}(t, q) \leq \mathcal{E}_{\varepsilon}(t, \check{q})+\mathcal{R}_{\varepsilon}(\check{z}-z) \forall \check{q} \in \mathcal{Q}\right\} .
$$

For $\varepsilon$ fixed, we shall say that a sequence $\left(t_{n}, q_{n}\right)_{n \in \mathbb{N}}$ is stable with respect to $\mathcal{E}_{\varepsilon}$ and $\mathcal{R}_{\varepsilon}$ if

$$
q_{n} \in \mathcal{S}_{\varepsilon}\left(t_{n}\right) \quad \text { and } \quad \sup _{n \in \mathbb{N}} \mathcal{E}_{\varepsilon}\left(t_{n}, q_{n}\right)<+\infty
$$

We shall say that a sequence $\left(t_{\varepsilon}, q_{\varepsilon}\right)$ is uniformly stable with respect to the collection $\left(\mathcal{E}_{\varepsilon}\right)_{\varepsilon>0}$ and $\left(\mathcal{R}_{\varepsilon}\right)_{\varepsilon>0}$ if

$$
q_{\varepsilon} \in \mathcal{S}_{\varepsilon}\left(t_{\varepsilon}\right) \quad \text { and } \quad \sup _{\varepsilon} \mathcal{E}_{\varepsilon}\left(t_{\varepsilon}, q_{\varepsilon}\right)<+\infty
$$

To be formal we should have put $\varepsilon_{n}$ in place of $\varepsilon$ everywhere in the definition above. Hence the notion of uniformly stable sequence depends on which sequence of functionals it is referred to. According to the mentioned shorthand convention, in the sequel we omit to state explicitly this reference when it can be easily deduced from the context.

\section{Delamination of the adhesive unilateral contact in $3 \mathrm{D}$ bodies}

In this section we consider a Fremond's [22-24] type model for delamination in 3D bodies which is the starting point of our asymptotic analysis and which has been discussed, for instance, in [62]. In particular, the existence of an energetic solution has been proven in [35] where also a numerical analysis of some model examples is performed. In [61] this model has been characterized as a modification (or, in fact, regularization) of the Griffith concept [26], based on the hypothesis that the two parts of the body can be delaminated just by a phenomenologically prescribed specific energy $a_{\varepsilon}$ (in physical units $\mathrm{J} / \mathrm{m}^{2}$ ).

For later purposes, we indicate the dependence on the thickness parameter $\varepsilon$, even if it will be considered fixed thoroughout this whole Section 2. Moreover, a delamination parameter $z: \Gamma_{\mathrm{C}} \rightarrow[0,1]$ is involved, representing the fraction of fixed adhesive: $z(x)=0$ means complete delamination, $z(x)=1$ means perfect integrity and, for instance, $z(x)=\frac{1}{2}$ means that $50 \%$ of the adhesive is debonded at $x \in \Gamma_{\mathrm{C}}$. This model is determined by the stored energy $\widetilde{\mathcal{E}}_{\varepsilon}$ and the dissipation energy $\mathcal{R}_{\varepsilon}$. The stored energy $\widetilde{\mathcal{E}}_{\varepsilon}$, depending on the time $t$, on the displacement $u$, and on the delamination parameter $z$, is given by

$$
\widetilde{\mathcal{E}}_{\varepsilon}(t, u, z)= \begin{cases}\frac{1}{2} \int_{\Omega_{1}^{\varepsilon} \cup \Omega_{2}^{\varepsilon}} \mathbb{C} e(u): e(u)-2 f^{\varepsilon}(t) \cdot u \mathrm{~d} x+\int_{\Gamma_{\mathrm{C}}} z Q^{\varepsilon}(\llbracket u \rrbracket) \mathrm{d} \mathscr{H}^{2} & \text { if }(u, z) \in \mathcal{A}_{\varepsilon}^{\mathrm{ad}}\left(w_{\mathrm{D}}^{\varepsilon}(t)\right), \\ +\infty & \text { else },\end{cases}
$$

where $e(u):=\frac{1}{2}(\nabla u)^{\top}+\frac{1}{2} \nabla u$ is the small-strain tensor and $\mathbb{C}$ is the tensor of elastic moduli which may depend on $x$ in the following way:

$$
\mathbb{C}=\mathbb{C}(x)= \begin{cases}\mathbb{C}^{(1)} & \text { if } x \in \Omega_{1} \\ \mathbb{C}^{(2)} & \text { if } x \in \Omega_{2}\end{cases}
$$

The symbol $\llbracket \cdot \rrbracket$ in $(2.1)$ denotes the jump across the surface $\Gamma_{\mathrm{C}}$ and $Q^{\varepsilon}: \mathbb{R}^{3} \rightarrow \mathbb{R}$ is a positive-definite quadratic form allowing for an anistropy of the adhesive to be specified later, cf. (3.6), and the admissible domain dependent on the prescribed Dirichlet condition $w_{\mathrm{D}}^{\varepsilon}$ is

$$
\mathcal{A}_{\varepsilon}^{\mathrm{ad}}\left(w_{\mathrm{D}}\right)=\left\{(u, z) \in W^{1,2}\left(\Omega_{1}^{\varepsilon} \cup \Omega_{2}^{\varepsilon} ; \mathbb{R}^{3}\right) \times L^{\infty}\left(\Gamma_{\mathrm{C}}\right): u=w_{\mathrm{D}} \text { on } \Gamma_{\mathrm{D}}^{\varepsilon}, \quad 0 \leq z \leq 1, \quad \llbracket u \rrbracket \cdot \nu \geq 0 \text { on } \Gamma_{\mathrm{C}}\right\}
$$

where $\nu$ is the normal to $\Gamma_{\mathrm{C}}$ (i.e., the unit vector of the axis $x_{3}$, see Fig.1). Finally,

$$
\mathcal{R}_{\varepsilon}(\dot{z}):= \begin{cases}\int_{\Gamma_{\mathrm{C}}} a_{\varepsilon}|\dot{z}| \mathrm{d} \mathscr{H}^{2} & \text { if } \dot{z} \leq 0 \text { on } \Gamma_{\mathrm{C}}, \\ +\infty & \text { else. }\end{cases}
$$


The $\varepsilon$-dependence of the body forces $f^{\varepsilon} \in C^{1}\left([0, T] ; L^{2}\left(\Omega^{\varepsilon} ; \mathbb{R}^{3}\right)\right)$ and the boundary displacements $w_{\mathrm{D}}^{\varepsilon} \in$ $C^{1}\left([0, T] ; H^{1 / 2}\left(\overline{\Gamma_{\mathrm{D}}^{\varepsilon}} ; \mathbb{R}^{3}\right)\right)$ will be precised in Section 3.1, cf. (3.3)-(3.5).

We assume that $\mathbb{C}^{(\ell)}$ are fourth-order positive-definite tensors, i.e.,

$$
\mathbb{C}^{(\ell)} e: e \geq c|e|^{2}, \quad \ell=1,2,
$$

for every symmetric matrix $e \in \mathbb{R}_{\mathrm{sym}}^{3 \times 3}$ and with some $c>0$ and that

$$
a_{\varepsilon}(x) \geq a_{\varepsilon, \min }
$$

for a suitable constant $a_{\varepsilon, \min }>0$ (depending on $\varepsilon$, but not on $x$ ) and $\mathscr{H}^{2}$-a.e. $x \in \Gamma_{\mathrm{C}}$. Moreover, we assume the following usual symmetry properties

$$
\mathbb{C}_{i j k l}^{(\ell)}=\mathbb{C}_{i j l k}^{(\ell)}=\mathbb{C}_{k l i j}^{(\ell)}, \quad i, j, k, l=1,2,3, \quad \ell=1,2,
$$

and that the material has a monoclinic symmetry with respect to the $\left(x_{1}, x_{2}\right)$-plane, which implies

$$
\mathbb{C}_{\alpha \beta \gamma 3}^{(\ell)}=\mathbb{C}_{\alpha 333}^{(\ell)}=0, \quad \alpha, \beta, \gamma=1,2, \quad \ell=1,2 .
$$

We restrict to the monoclinic symmetry for simplicity. In the absence of delamination, under this assumption, the limit problem decouples into two separate problems: one consisting in the in-plane equilibrium equations and the other for the out-of-plane equations.

It should be noted that Definition 1.1 does not apply directly to the problem involving $\widetilde{\varepsilon}_{\varepsilon, \kappa}$ if the Dirichlet loading $w_{\mathrm{D}}^{\varepsilon}$ varies in time because the time derivative in $(1.4 \mathrm{~b})$ is not well defined. To handle it, one must transform the problem into a time-independent Dirichlet loading problem. In this way, we consider a prolongation $u_{\mathrm{D}}^{\varepsilon}(t)$ of $w_{\mathrm{D}}^{\varepsilon}(t)$ to the whole $\Omega^{\varepsilon}$ satisfying

$$
\llbracket u_{\mathrm{D}}^{\varepsilon}(t) \rrbracket=0 \quad \text { a.e. on } \Gamma_{\mathrm{C}} \text { for a.a. } t,
$$

and then, instead of (2.1), we consider

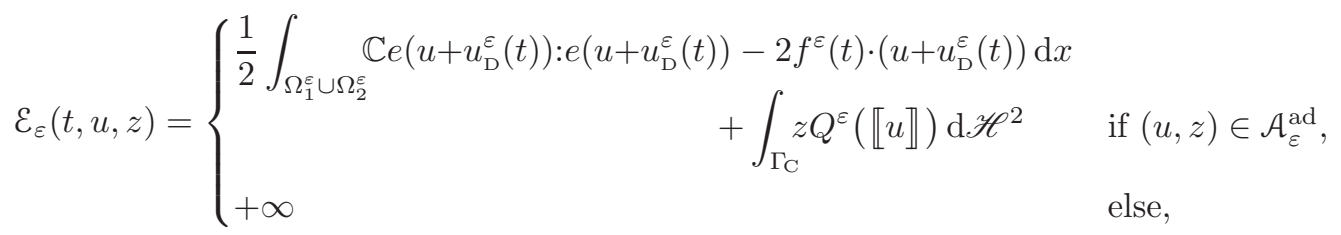

where $\mathcal{A}_{\varepsilon}^{\text {ad }}:=\mathcal{A}_{\varepsilon}^{\text {ad }}(0)$. The spaces $\mathcal{U}, \mathcal{Z}$ and $\mathcal{Q}$ in Definition 1.1 are as

$$
\mathcal{U}_{\varepsilon}=\left\{u \in W^{1,2}\left(\Omega_{1}^{\varepsilon} \cup \Omega_{2}^{\varepsilon} ; \mathbb{R}^{3}\right): u=0 \text { on } \Gamma_{\mathrm{D}}^{\varepsilon}\right\}, \mathcal{Z}=\left\{z \in L^{\infty}\left(\Gamma_{\mathrm{C}}\right): 0 \leq z \leq 1 \text { on } \Gamma_{\mathrm{C}}\right\}, Q=\mathcal{U}_{\varepsilon} \times \mathcal{Z} ;
$$

here both $\mathfrak{U}_{\varepsilon}$ and $z$ are even subsets of Banach spaces and we will consider them equipped with standard norm, weak, and weak* topologies. Having an energetic solution $\left(u_{\varepsilon}, z_{\varepsilon}\right)$ to the problem $\left(\mathcal{U}_{\varepsilon} \times \mathcal{Z}_{\varepsilon}, \mathcal{E}_{\varepsilon}, \mathcal{R}_{\varepsilon}, q^{0}\right)$ according to Definition 1.1, the shifted solution $\left(u_{\varepsilon}+u_{\mathrm{D}}^{\varepsilon}, z_{\varepsilon}\right)$ will serve as an energetic solution to the original problem involving $\widetilde{\mathcal{E}}_{\varepsilon}$. We will thus deal only with the transformed problems. Note that here it is desirable to accept that $\Gamma_{\mathrm{C}}$ and $\Gamma_{\mathrm{D}}^{\varepsilon}$ are not far from each other (i.e., their closures may not be disjoint), which requires the compatibility of the boundary condition (2.8). More specifically, we assume

$$
w_{\mathrm{D}}^{\varepsilon} \in C^{1}\left([0, T] ; H^{1 / 2}\left(\overline{\Gamma_{\mathrm{D}}^{\varepsilon}} ; \mathbb{R}^{3}\right)\right),
$$

which, in particular, ensures the existence of a prolongation $u_{\mathrm{D}}^{\varepsilon} \in C^{1}\left([0, T] ; W^{1,2}\left(\overline{\Omega^{\varepsilon}} ; \mathbb{R}^{3}\right)\right)$ satisfying $(2.8)$ for all $t$.

The following proposition can be proven by applying general existence results on energetic solutions, cf. [46,51], and of course follows also by [35, Proposition 3.3].

Proposition 2.1. Let $\varepsilon>0$ be fixed, assume (2.11), and let $q^{0}=\left(u^{0}, z^{0}\right)$ be stable at time $t=0$, that is

$$
\mathcal{E}_{\varepsilon}\left(0, q^{0}\right)<+\infty \quad \& \quad \mathcal{E}_{\varepsilon}\left(0, q^{0}\right) \leq \mathcal{E}_{\varepsilon}(0, \check{q})+\mathcal{R}_{\varepsilon}\left(\check{z}-z^{0}\right) \quad \text { for all } \check{q}=(\check{u}, \check{z}) \in \mathcal{Q} .
$$

Then the energetic solution $\left(u_{\varepsilon}, z_{\varepsilon}\right)$ to the problem $\left(\mathcal{U}_{\varepsilon} \times \mathcal{Z}, \mathcal{E}_{\varepsilon}, \mathcal{R}_{\varepsilon}, q^{0}\right)$ does exist. 


\section{Dimension reduction}

In this section, we rescale the family of the problems on the domains $\Omega_{1}^{\varepsilon} \cup \Omega_{2}^{\varepsilon}$ parameterized by $\varepsilon>0$ to a single auxiliary domain arising for $\varepsilon=1$, denoted by $\Omega_{1} \cup \Omega_{2}$, and analyze the convergence as $\varepsilon \rightarrow 0$.

\subsection{The rescaled problem}

To perform the dimension reduction it is convenient to make a change of variables in order to work on domains independent of $\varepsilon$. There are lot of similarities with [21, Sect.3-5] so that we expose it only briefly. Beside $\Omega_{1}:=\Omega_{1}^{1}, \Omega_{2}:=\Omega_{2}^{1}$, let also $\Gamma_{\mathrm{D} 1}:=\Gamma_{\mathrm{D} 1}^{1}$ and $\Gamma_{\mathrm{D} 2}:=\Gamma_{\mathrm{D} 2}^{1}$, so that $\Omega:=\Omega^{1}$ and $\Gamma_{\mathrm{D}}:=\Gamma_{\mathrm{D}}^{1}$. For any $\varepsilon>0$, let $p_{\varepsilon}: \overline{\Omega_{1} \cup \Omega_{2}} \rightarrow \overline{\Omega_{1}^{\varepsilon} \cup \Omega_{2}^{\varepsilon}}$ be defined by

$$
p_{\varepsilon}\left(x_{1}, x_{2}, x_{3}\right):=\left(x_{1}, x_{2}, \varepsilon x_{3}\right)
$$

The variables on the fixed domain, $\Omega_{1} \cup \Omega_{2}$, will be denoted by using the Sans font. Thus for $u \in$ $W^{1,2}\left(\Omega_{1}^{\varepsilon} \cup \Omega_{2}^{\varepsilon} ; \mathbb{R}^{3}\right)$, let $\mathrm{u} \in W^{1,2}\left(\Omega_{1} \cup \Omega_{2} ; \mathbb{R}^{3}\right)$ be defined by

$$
\begin{aligned}
\mathrm{u}_{\alpha} & :=\frac{1}{\varepsilon} u_{\alpha} \circ p_{\varepsilon}, \alpha=1,2, \\
\mathrm{u}_{3} & :=u_{3} \circ p_{\varepsilon} .
\end{aligned}
$$

Of course, $z$ is not scaled and thus it remains in Italic font. With this notation we have

$$
\frac{1}{\varepsilon} e(u) \circ p_{\varepsilon}=\left(\begin{array}{cc}
e(\mathbf{u})_{\alpha \beta} & \frac{1}{\varepsilon} e(\mathbf{u})_{\alpha 3} \\
\frac{1}{\varepsilon} e(\mathbf{u})_{3 \beta} & \frac{1}{\varepsilon^{2}} e(\mathbf{u})_{33}
\end{array}\right)=: e^{\varepsilon}(\mathbf{u}) .
$$

In order to keep the displacements bounded, we need to rescale the forces and the boundary conditions with $\varepsilon$. We assume that there exists $\mathrm{f} \in C^{1}\left([0, T] ; L^{2}\left(\Omega ; \mathbb{R}^{3}\right)\right)$ such that

$$
\varepsilon^{\ell_{\tau}+1} \mathrm{f}_{\alpha}(t)=f_{\alpha}^{\varepsilon}(t) \circ p_{\varepsilon}, \alpha=1,2, \quad \text { and } \quad \varepsilon^{\ell_{\nu}+2} \mathrm{f}_{3}(t)=f_{3}^{\varepsilon}(t) \circ p_{\varepsilon}, \quad \ell_{\tau}, \ell_{\nu} \geq 0 .
$$

Concerning the boundary conditions we need to require that $e^{\varepsilon}\left(\mathbf{u}_{\mathrm{D}}^{\varepsilon}(t)\right)$ is bounded in $L^{2}\left(\Omega_{1} \cup \Omega_{2} ; \mathbb{R}^{3 \times 3}\right)$, where we have set $\left[u_{\mathrm{D}}^{\varepsilon}\right]_{\alpha}:=\frac{1}{\varepsilon}\left[u_{\mathrm{D}}^{\varepsilon}\right]_{\alpha} \circ p_{\varepsilon}$ and $\left[u_{\mathrm{D}}^{\varepsilon}\right]_{3}:=\left[u_{\mathrm{D}}^{\varepsilon}\right]_{3} \circ p_{\varepsilon}$. The simplest way to fulfill this requirement is to pose $u_{D}^{\varepsilon}=u_{D}$, with $u_{D}$ satisfying the following Kirchhoff-Love assumption which is fundamental in plate theory, cf. [11]:

$$
\begin{aligned}
& {\left[\mathrm{u}_{\mathrm{D}}\right]_{\alpha}\left(t, x_{1}, x_{2}, x_{3}\right):=\left[\rho_{\mathrm{D}}\right]_{\alpha}\left(t, x_{1}, x_{2}\right)-x_{3} \frac{\partial}{\partial x_{\alpha}} \xi_{\mathrm{D}}\left(t, x_{1}, x_{2}\right), \quad \alpha=1,2,} \\
& {\left[\mathrm{u}_{\mathrm{D}}\right]_{3}\left(t, x_{1}, x_{2}, x_{3}\right):=\xi_{\mathrm{D}}\left(t, x_{1}, x_{2}\right),}
\end{aligned}
$$

where $\xi_{\mathrm{D}} \in C^{1}\left([0, T] ; W^{2,2}(\Omega)\right)$ and $\rho_{\mathrm{D}} \in C^{1}\left([0, T] ; W^{1,2}\left(\Omega ; \mathbb{R}^{2}\right)\right)$. Let us remark that with this choice we have

$$
e^{\varepsilon}\left(\mathrm{u}_{\mathrm{D}}^{\varepsilon}\right)_{i 3}=0, \quad i=1,2,3,
$$

hence, in particular, $e^{\varepsilon}\left(\mathbf{u}_{\mathrm{D}}^{\varepsilon}\right)=e^{\varepsilon}\left(\mathbf{u}_{\mathrm{D}}\right)=e\left(\mathbf{u}_{\mathrm{D}}\right)$ for any $\varepsilon$.

The quadratic form used in (2.1), possibly scaling anisotropically the elastic response of the adhesive, is considered, for any $\varepsilon>0$, to be of the form, see for instance Tavara et al. [63],

$$
Q^{\varepsilon}(s):=\varepsilon^{q_{\tau}+1} \kappa_{\tau}\left|s_{\tau}\right|^{2}+\varepsilon^{q_{\nu}+3} \kappa_{\nu}\left|s_{\nu}\right|^{2}, \quad q_{\tau}, q_{\nu} \in \mathbb{R}, \kappa_{\tau}, \kappa_{\nu}>0,
$$

where $s=\left(s_{\tau}, s_{\nu}\right) \in \mathbb{R}^{2} \times \mathbb{R}$.

Denoting by $\llbracket \mathrm{u} \rrbracket_{\tau}:=\left(\llbracket \mathrm{u}_{1} \rrbracket, \llbracket \mathrm{u}_{2} \rrbracket\right)$ and $\llbracket \mathrm{u} \rrbracket_{\nu}:=\llbracket \mathrm{u}_{3} \rrbracket$, respectively, the tangential and the normal jump of the displacement across the delamination surface $\Gamma_{\mathrm{C}}$, and using the relation (3.1) between $u \in W^{1,2}\left(\Omega_{1}^{\varepsilon} \cup\right.$ $\left.\Omega_{2}^{\varepsilon} ; \mathbb{R}^{3}\right)$ and $\mathrm{u} \in W^{1,2}\left(\Omega_{1} \cup \Omega_{2} ; \mathbb{R}^{3}\right)$, for any $\varepsilon>0$ we get

$$
Q^{\varepsilon}(\llbracket u \rrbracket)=\varepsilon^{q_{\tau}+3} \kappa_{\tau}\left|\llbracket \mathrm{u} \rrbracket \rrbracket_{\tau}\right|^{2}+\varepsilon^{q_{\nu}+3} \kappa_{\nu}\left|\llbracket \mathrm{u} \rrbracket_{\nu}\right|^{2}=\varepsilon^{3} \mathrm{Q}^{\varepsilon}(\llbracket \mathrm{u} \rrbracket)
$$


having defined

$$
\mathrm{Q}^{\varepsilon}(\llbracket \mathrm{u} \rrbracket):=\varepsilon^{q_{\tau}} \kappa_{\tau}\left|\llbracket \mathrm{u} \rrbracket_{\tau}\right|^{2}+\varepsilon^{q_{\nu}} \kappa_{\nu}\left|\llbracket \mathrm{u} \rrbracket_{\nu}\right|^{2} .
$$

The functional $\mathrm{Q}^{\varepsilon}$ (as well as $Q^{\varepsilon}$ ) modulates differently the components of the jump of u. The different scalings take into account the different rigidity in the in-plane and in the out-of-plane directions of the cylinders of height $\varepsilon h_{\ell}, \ell=1,2$.

It is convenient to do the same also with the body force. Recalling (3.3) and using again (3.1) we observe that

$$
f^{\varepsilon} \cdot\left(u+u_{\mathrm{D}}^{\varepsilon}\right)=\sum_{\alpha=1,2} \varepsilon^{\ell_{\tau}+2} \mathrm{f}_{\alpha}^{\varepsilon}\left(\mathrm{u}_{\alpha}+\left[\mathrm{u}_{\mathrm{D}}\right]_{\alpha}\right)+\varepsilon^{\ell_{\nu}+2} \mathrm{f}_{3}^{\varepsilon}\left(\mathrm{u}_{3}+\left[\mathrm{u}_{\mathrm{D}}\right]_{3}\right)=: \varepsilon^{2} \mathrm{f}^{\varepsilon} \cdot\left(\mathrm{u}+\mathrm{u}_{\mathrm{D}}\right),
$$

having defined

For $(u, z) \in \mathcal{A}_{\varepsilon}^{\text {ad }}$ we have

$$
\mathbf{f}^{\varepsilon}:=\left(\varepsilon^{\ell_{\tau}} f_{1}, \varepsilon^{\ell_{\tau}} f_{2}, \varepsilon^{\ell_{\nu}} f_{3}\right)
$$

$$
\begin{aligned}
\mathcal{E}_{\varepsilon}(t, u, z) & =\frac{1}{2} \int_{\Omega_{1}^{\varepsilon} \cup \Omega_{2}^{\varepsilon}} \mathbb{C} e\left(u+u_{\mathrm{D}}^{\varepsilon}(t)\right): e\left(u+u_{\mathrm{D}}^{\varepsilon}(t)\right)-2 f^{\varepsilon}(t) \cdot\left(u+u_{\mathrm{D}}^{\varepsilon}(t)\right) \mathrm{d} x+\int_{\Gamma_{\mathrm{C}}} z Q^{\varepsilon}(\llbracket u \rrbracket) \mathrm{d} \mathscr{H}^{2} \\
& =\frac{\varepsilon^{3}}{2} \int_{\Omega_{1} \cup \Omega_{2}} \mathbb{C} e^{\varepsilon}\left(\mathbf{u}+\mathrm{u}_{\mathrm{D}}(t)\right): e^{\varepsilon}\left(\mathbf{u}+\mathrm{u}_{\mathrm{D}}(t)\right)-2 \mathrm{f}^{\varepsilon}(t) \cdot\left(\mathbf{u}+\mathrm{u}_{\mathrm{D}}(t)\right) \mathrm{d} x+\varepsilon^{3} \int_{\Gamma_{\mathrm{C}}} z \mathrm{Q}^{\varepsilon}(\llbracket \mathbf{u} \rrbracket) \mathrm{d} \mathscr{H}^{2} \\
& =\varepsilon^{3} \mathrm{E}_{\varepsilon}(t, \mathbf{u}, z),
\end{aligned}
$$

where we used

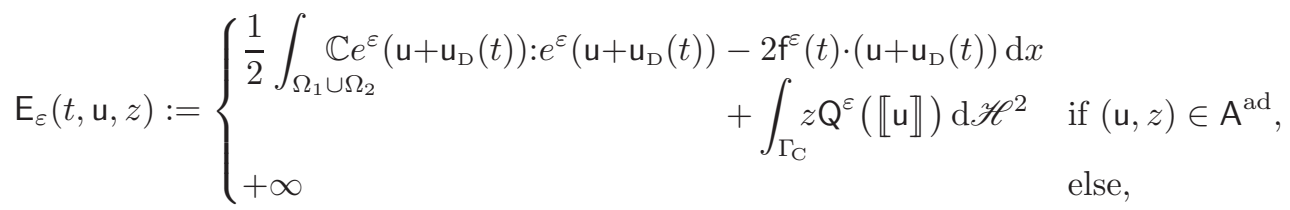

with

$$
\mathrm{A}^{\mathrm{ad}}:=\left\{(\mathrm{u}, z) \in W^{1,2}\left(\Omega_{1} \cup \Omega_{2} ; \mathbb{R}^{3}\right) \times L^{\infty}\left(\Gamma_{\mathrm{C}}\right): \mathrm{u}=0 \text { on } \Gamma_{\mathrm{D}}, \quad 0 \leq z \leq 1, \quad \llbracket \mathrm{u} \rrbracket_{\nu} \geq 0 \text { on } \Gamma_{\mathrm{C}}\right\} .
$$

The energy functional has been scaled by dividing by $\varepsilon^{3}$, as it is conventionally done in linear elasticity. On the other hand, it is easy to see that any other choice turns out to be equivalent. Indeed, since the bulk energy is a quadratic form of $u$, one can always reduce to this case by suitably re-scaling the displacements. Similarly we rescale the dissipation energy; we assume that there exist a measurable function $\mathrm{a} \in L^{1}\left(\Gamma_{\mathrm{C}}\right)$ and $d \in \mathbb{R}$ such that

$$
\frac{a_{\varepsilon}}{\varepsilon^{3+d}}=\mathrm{a}
$$

for any $\varepsilon$, so that

$$
\mathcal{R}_{\varepsilon}(\dot{z})=\varepsilon^{3} \mathrm{R}_{\varepsilon}^{d}(\dot{z})
$$

where

$$
\mathrm{R}_{\varepsilon}^{d}(\dot{z}):= \begin{cases}\varepsilon^{d} \int_{\Gamma_{\mathrm{C}}} \mathrm{a}|\dot{z}| \mathrm{d} \mathscr{H}^{2} & \text { if } \dot{z} \leq 0 \text { on } \Gamma_{\mathrm{C}} \\ +\infty & \text { else. }\end{cases}
$$

The choice $d=0$ is the one that yields the same scaling of the stored energy, and only in this case we expect to obtain a non-trivial dissipation in the limit problem (see Liero and Mielke [43, Remark 2.1] for a justification "a priori" of this choice). Referring to (2.5), we also assume that $a_{\varepsilon, \min }=\varepsilon^{3+d} \mathrm{a}_{\min }$ for a suitable constant $\mathrm{a}_{\min }>0$ so that $\mathrm{a}(x) \geq \mathrm{a}_{\min }$ for $\mathscr{H}^{2}$-a.e. $x \in \Gamma_{\mathrm{C}}$.

To conclude this section, it is worth notice that the dissipation energy $\mathrm{R}_{\varepsilon}^{d}$ induces the following dissipation distance

$$
\mathcal{D}_{\varepsilon}^{d}\left(z_{1}, z_{2}\right):=\mathrm{R}_{\varepsilon}^{d}\left(z_{2}-z_{1}\right)
$$

Moreover, concerning the notation used in Definition 1.1, we have that $\operatorname{Diss}_{\mathrm{R}_{\varepsilon}^{d}}(z,[0, t])=\mathcal{D}_{\varepsilon}^{d}(z(0)-z(t))$. 


\subsection{The limit problem}

It is further useful to introduce the following "reduced" tensor of elastic moduli

$$
\mathbb{C}_{\alpha \beta \gamma \delta}^{0}:=\mathbb{C}_{\alpha \beta \gamma \delta}-\frac{\mathbb{C}_{\alpha \beta 33} \mathbb{C}_{\gamma \delta 33}}{\mathbb{C}_{3333}}, \quad \alpha, \beta, \gamma, \delta=1,2 .
$$

Let us remark (see also [1]) that, when setting $g(e):=\frac{1}{2} \mathbb{C} e: e$ for $e \in \mathbb{R}_{\mathrm{sym}}^{3 \times 3}$, under assumptions (2.6) and (2.7) one has

$$
\frac{1}{2} \mathbb{C}^{0} \tilde{e}: \tilde{e}=\min _{\eta \in \mathbb{R}^{2}, \rho_{3} \in \mathbb{R}} g\left(\begin{array}{cc}
\tilde{e} & \eta \\
\eta^{\top} & \rho_{3}
\end{array}\right) \quad \text { for every } \tilde{e} \in \mathbb{R}_{\mathrm{sym}}^{2 \times 2},
$$

where the minimum is achieved for $\eta=0$ and $\rho_{3}=-\sum_{\alpha, \beta=1}^{2} \mathbb{C}_{33 \alpha \beta} \tilde{e}_{\alpha \beta} / \mathbb{C}_{3333}$. Of course, $\mathbb{C}^{0}$ depends on $x_{3}$ as $\mathbb{C}$ does, cf. (2.2). Moreover, let us denote by $\tilde{e}(\mathrm{u})$ the $2 \times 2$-matrix with components

$$
\tilde{e}(\mathbf{u})_{\alpha \beta}:=e(\mathbf{u})_{\alpha \beta}, \quad \alpha, \beta=1,2 .
$$

Before we can write the expected limit energy, it remains to introduce the quadratic form

$$
\mathrm{Q}^{0}(s):=1_{0}\left(q_{\tau}\right) \kappa_{\tau}\left|s_{\tau}\right|^{2}+1_{0}\left(q_{\nu}\right) \kappa_{\nu}\left|s_{\nu}\right|^{2}, \quad s \in \mathbb{R}^{2} \times \mathbb{R}
$$

and the vector field

$$
\mathrm{f}^{0}:=\left(1_{0}\left(\ell_{\tau}\right) \mathrm{f}_{1}, 1_{0}\left(\ell_{\tau}\right) \mathrm{f}_{2}, 1_{0}\left(\ell_{\nu}\right) \mathrm{f}_{3}\right)
$$

where $1_{0}(r)$ takes the value 1 if $r=0$ and is 0 otherwise. Let us remark that the choice $q_{\tau}=q_{\nu}=0$, $\kappa_{\tau}=\kappa_{\nu}=\kappa$ gives $\mathrm{Q}^{0}(\llbracket \mathrm{u} \rrbracket)=\kappa|\llbracket \mathrm{u} \rrbracket|^{2}$.

Then we define

$$
\mathrm{E}_{0}(t, \mathrm{u}, z):=\left\{\begin{array}{cl}
\frac{1}{2} \int_{\Omega_{1} \cup \Omega_{2}} \mathbb{C}^{0} \tilde{e}\left(\mathrm{u}+\mathrm{u}_{\mathrm{D}}(t)\right): \tilde{e}\left(\mathbf{u}+\mathrm{u}_{\mathrm{D}}(t)\right) & \\
-2 \mathrm{f}^{0}(t) \cdot\left(\mathrm{u}+\mathrm{u}_{\mathrm{D}}(t)\right) \mathrm{d} x+\int_{\Gamma_{\mathrm{C}}} z \mathrm{Q}^{0}(\llbracket \mathrm{u} \rrbracket) \mathrm{d} \mathscr{H}^{2} & \text { if }(\mathrm{u}, z) \in \mathrm{A}_{\mathrm{KL}}^{\mathrm{ad}}, \\
+\infty & \text { else },
\end{array}\right.
$$

where

$$
\mathrm{A}_{\mathrm{KL}}^{\mathrm{ad}}=\left\{(\mathrm{u}, z) \in \mathrm{A}^{\mathrm{ad}}: \mathrm{u} \in W_{\mathrm{KL}}^{1,2}\left(\Omega_{1} \cup \Omega_{2} ; \mathbb{R}^{3}\right) \text { and } z \llbracket \mathrm{u} \rrbracket_{\sigma}=0 \text { a.e. on } \Gamma_{\mathrm{C}} \text { if } q_{\sigma}<0(\sigma=\tau, \nu)\right\},
$$

and where $W_{\mathrm{KL}}^{1,2}\left(\Omega_{1} \cup \Omega_{2} ; \mathbb{R}^{3}\right)$ stands for the space of Kirchhoff-Love displacements

$$
W_{\mathrm{KL}}^{1,2}\left(\Omega_{1} \cup \Omega_{2} ; \mathbb{R}^{3}\right):=\left\{\mathrm{u} \in W^{1,2}\left(\Omega_{1} \cup \Omega_{2} ; \mathbb{R}^{3}\right): e(\mathrm{u})_{i 3}=0 \text { for } i=1,2,3\right\} .
$$

Finally, we define

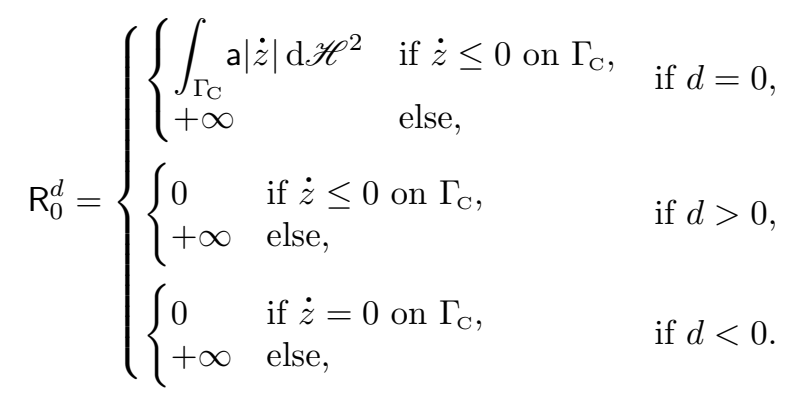

The evolution problems will be considered on the spaces

$$
\mathcal{U}=\left\{\mathrm{u} \in W^{1,2}\left(\Omega_{1} \cup \Omega_{2} ; \mathbb{R}^{3}\right): \mathrm{u}=0 \text { on } \Gamma_{\mathrm{D}}\right\} \quad \text { and } \quad z \text { from }(2.10) .
$$




\subsection{Convergence}

We discuss here the convergence of energetic solutions associated with the problem $\left(\mathcal{U} \times \mathcal{Z}, \mathrm{E}_{\varepsilon}, \mathrm{R}_{\varepsilon}^{d}, \mathrm{q}_{0, \varepsilon}\right)$ to energetic solutions associated to the problem $\left(\mathcal{U} \times \mathcal{Z}, \mathrm{E}_{0}, \mathrm{R}_{0}^{d}, q_{0}\right)$, as $\varepsilon \rightarrow 0$. This is done by applying the abstract scheme developed in [50]. Since most of the requirements can be obtained as simple adaptations from [21], we mainly highlight the differences.

The closure of $A^{\text {ad }}$ and of the constraints on the jumps, the continuity of the functional representing the work of the body forces, the convergence of the powers

$$
\partial_{t} \mathrm{E}_{\varepsilon}\left(t_{\varepsilon}, \mathbf{u}_{\varepsilon}, z_{\varepsilon}\right)=\int_{\Omega_{1} \cup \Omega_{2}} \mathbb{C} e^{\varepsilon}\left(\dot{u}_{\mathrm{D}}\left(t_{\varepsilon}\right)\right): e^{\varepsilon}\left(\mathbf{u}_{\varepsilon}+\mathrm{u}_{\mathrm{D}}\left(t_{\varepsilon}\right)\right)-\dot{f}^{\varepsilon}\left(t_{\varepsilon}\right) \cdot\left(\mathrm{u}_{\varepsilon}+\mathrm{u}_{\mathrm{D}}\left(t_{\varepsilon}\right)\right)-\mathrm{f}^{\varepsilon}\left(t_{\varepsilon}\right) \cdot \dot{\mathrm{u}}_{\mathrm{D}}\left(t_{\varepsilon}\right) \mathrm{d} x
$$

to

$$
\partial_{t} \mathrm{E}_{0}(t, \mathbf{u}, z)=\int_{\Omega_{1} \cup \Omega_{2}} \mathbb{C}^{0} \tilde{e}\left(\dot{\mathrm{u}}_{\mathrm{D}}(t)\right): \tilde{e}\left(\mathbf{u}+\mathbf{u}_{\mathrm{D}}(t)\right)-\dot{\mathrm{f}}^{0}(t) \cdot\left(\mathbf{u}+\mathrm{u}_{\mathrm{D}}(t)\right)-\mathrm{f}^{0}(t) \cdot \dot{\mathrm{u}}_{\mathrm{D}}(t) \mathrm{d} x,
$$

provided that $t_{\varepsilon} \rightarrow t, z_{\varepsilon} \stackrel{*}{\rightarrow} z$ in $L^{\infty}\left(\Gamma_{\mathrm{C}}\right), 0 \leq z_{\varepsilon} \leq 1$ a.e., and $\mathrm{u}_{\varepsilon} \rightarrow \mathrm{u}$ in $W^{1,2}\left(\Omega_{1} \cup \Omega_{2} ; \mathbb{R}^{3}\right)$ as $\varepsilon \rightarrow 0^{+}$, with $\left(t_{\varepsilon}, \mathrm{u}_{\varepsilon}, z_{\varepsilon}\right)$ being a uniformly stable sequence (cf. (1.7) for $\mathrm{E}_{\varepsilon}$ and $\mathrm{R}_{\varepsilon}^{d}$ instead of $\mathcal{E}_{\varepsilon}$ and $\mathcal{R}_{\varepsilon}$, respectively) follow by a straightforward adaptation of the proofs of [21, Lemmas 4.1 and 5.2] concerning the case of a transversal delamination surface.

In the next lemma we prove the lower semicontinuity inequality (3.21a) and the existence of a so-called mutual recovery sequence, since these results are not simple adaptations of arguments of [21].

Lemma 3.1. If $t_{\varepsilon} \rightarrow t, z_{\varepsilon} \stackrel{*}{\rightarrow} z$ in $L^{\infty}\left(\Gamma_{\mathrm{C}}\right), 0 \leq z_{\varepsilon} \leq 1$ a.e., and $\mathrm{u}_{\varepsilon} \rightarrow \mathrm{u}$ in $W^{1,2}\left(\Omega_{1} \cup \Omega_{2} ; \mathbb{R}^{3}\right)$ as $\varepsilon \rightarrow 0^{+}$, then

$$
\liminf _{\varepsilon \rightarrow 0^{+}} \mathrm{E}_{\varepsilon}\left(t_{\varepsilon}, \mathrm{u}_{\varepsilon}, z_{\varepsilon}\right) \geq \mathrm{E}_{0}(t, \mathrm{u}, z)
$$

and, for every $\check{z} \in L^{\infty}\left(\Gamma_{\mathrm{C}}\right)$ and every $\check{u} \in W^{1,2}\left(\Omega_{1} \cup \Omega_{2} ; \mathbb{R}^{3}\right)$, there exist $\check{z}_{\varepsilon} \in \mathcal{Z}$ and $\check{\mathrm{u}}_{\varepsilon} \in \mathcal{U}$ such that $\check{u}_{\varepsilon} \rightarrow$ ú in $W^{1,2}\left(\Omega_{1} \cup \Omega_{2} ; \mathbb{R}^{3}\right), \check{z}_{\varepsilon} \stackrel{*}{\rightarrow} \check{z}$ in $L^{\infty}\left(\Gamma_{\mathrm{C}}\right)$ and

$$
\limsup _{\varepsilon \rightarrow 0}\left[\mathrm{E}_{\varepsilon}\left(t_{\varepsilon}, \check{\mathrm{u}}_{\varepsilon}, \check{z}_{\varepsilon}\right)+\mathrm{R}_{\varepsilon}^{d}\left(\check{z}_{\varepsilon}-z_{\varepsilon}\right)\right] \leq \mathrm{E}_{0}(t, \check{\mathrm{u}}, \check{z})+\mathrm{R}_{0}^{d}(\check{z}-z) .
$$

Proof. We first observe that from the convergence assumptions on $\left(z_{\varepsilon}\right)$ and $\left(\mathbf{u}_{\varepsilon}\right)$, where the latter implies the strong convergence of the traces of $u_{\varepsilon}$ on $\Gamma_{\mathrm{C}}$, it follows that

$$
\int_{\Gamma_{\mathrm{C}}} z_{\varepsilon}\left|\llbracket \mathrm{u}_{\varepsilon} \rrbracket_{\sigma}\right|^{2} \mathrm{~d} \mathscr{H}^{2} \rightarrow \int_{\Gamma_{\mathrm{C}}} z\left|\llbracket \mathrm{u} \rrbracket_{\sigma}\right|^{2} \mathrm{~d} \mathscr{H}^{2}, \quad \sigma=\tau, \nu
$$

Let us prove the lower semicontinuity inequality (3.21a). Under the assumption that the liminf on the left-hand side be finite, by the positive definiteness of $\mathbb{C}$ (see (2.4)) and the continuity of $w$ with respect to $t$ and the fact that $\ell_{\tau}, \ell_{\nu} \geq 0$, there exists a positive constant $C$ such that

$$
\left\|e^{\varepsilon}\left(\mathbf{u}_{\varepsilon}\right)\right\|_{L^{2}\left(\Omega_{1} \cup \Omega_{2} ; \mathbb{R}^{3}\right)} \leq C
$$

and

$$
\int_{\Gamma_{\mathrm{C}}} z_{\varepsilon} \varepsilon^{q_{\sigma}}\left|\llbracket \mathrm{u}_{\varepsilon} \rrbracket_{\sigma}\right|^{2} \mathrm{~d} \mathscr{H}^{2} \leq C, \quad \sigma=\tau, \nu .
$$

For any $i=1,2,3$, from (3.23) we get $\left\|e\left(\mathrm{u}_{\varepsilon}\right)_{i 3}\right\|_{L^{2}\left(\Omega_{1} \cup \Omega_{2} ; \mathbb{R}^{3}\right)} \leq C \varepsilon$, thus, passing to the limit, we obtain $e(\mathrm{u})_{i 3}=0$, which implies $\mathbf{u} \in W_{\mathrm{KL}}^{1,2}\left(\Omega_{1} \cup \Omega_{2} ; \mathbb{R}^{3}\right)$.

Rewriting (3.24) as

$$
0 \leq \int_{\Gamma_{\mathrm{C}}} z_{\varepsilon}\left|\llbracket \mathrm{u}_{\varepsilon} \rrbracket_{\sigma}\right|^{2} \mathrm{~d} \mathscr{H}^{2} \leq C \varepsilon^{-q_{\sigma}},
$$

if $q_{\sigma}<0$, taking the limit as $\varepsilon \rightarrow 0^{+}$in this inequality we get

$$
\int_{\Gamma_{\mathrm{C}}} z\left|\llbracket \mathrm{u} \rrbracket_{\sigma}\right|^{2} \mathrm{~d} \mathscr{H}^{2}=0
$$


hence $z \llbracket \mathrm{u} \rrbracket_{\sigma}=0 \mathscr{H}^{2}$-a.e. on $\Gamma_{\mathrm{C}}$. Namely, $(u, z) \in \mathrm{A}_{\mathrm{KL}}^{\text {ad }}$ and therefore we have that also the right-hand side in (3.21a) is finite. Using (3.22) in the terms occurring in $Q^{\varepsilon}$ with $q_{\sigma}=0(\sigma=\tau, \nu)$ and neglecting those in which $q_{\sigma} \neq 0$, and using moreover the fact that

$$
\lim _{\varepsilon \rightarrow 0^{+}} \int_{\Omega_{1} \cup \Omega_{2}} f^{\varepsilon}(t) \cdot\left(u_{\varepsilon}+u_{D}(t)\right) d x=\int_{\Omega_{1} \cup \Omega_{2}} f^{0}(t) \cdot\left(u+u_{D}(t)\right) d x,
$$

we argue that it suffices to prove that

$$
\liminf _{\varepsilon \rightarrow 0^{+}} \int_{\Omega_{1} \cup \Omega_{2}} \mathbb{C} e^{\varepsilon}\left(\mathbf{u}_{\varepsilon}+\mathbf{u}_{\mathrm{D}}\left(t_{\varepsilon}\right)\right): e^{\varepsilon}\left(\mathbf{u}_{\varepsilon}+\mathbf{u}_{\mathrm{D}}\left(t_{\varepsilon}\right)\right) \mathrm{d} x \geq \int_{\Omega_{1} \cup \Omega_{2}} \mathbb{C}^{0} \tilde{e}\left(\mathbf{u}+\mathbf{u}_{\mathrm{D}}(t)\right): \tilde{e}\left(\mathbf{u}+\mathbf{u}_{\mathrm{D}}(t)\right) \mathrm{d} x .
$$

Since, for $\alpha, \beta=1,2$,

$$
e^{\varepsilon}\left(\mathbf{u}_{\varepsilon}\right)_{\alpha \beta}=e\left(\mathbf{u}_{\varepsilon}\right)_{\alpha \beta} \rightarrow e(\mathbf{u})_{\alpha \beta} \quad \text { in } L^{2}\left(\Omega_{1} \cup \Omega_{2} ; \mathbb{R}^{3}\right),
$$

using property (3.14) and the continuity of $\mathrm{u}_{\mathrm{D}}$ with respect to $t$ we find

$$
\begin{aligned}
\liminf _{\varepsilon \rightarrow 0^{+}} \int_{\Omega_{1} \cup \Omega_{2}} \mathbb{C} e^{\varepsilon}\left(\mathbf{u}_{\varepsilon}+\mathbf{u}_{\mathbf{D}}\left(t_{\varepsilon}\right)\right): e^{\varepsilon}\left(\mathbf{u}_{\varepsilon}+\mathbf{u}_{\mathbf{D}}\left(t_{\varepsilon}\right)\right) \mathrm{d} x & \geq \liminf _{\varepsilon \rightarrow 0^{+}} \int_{\Omega_{1} \cup \Omega_{2}} \mathbb{C} \tilde{e}\left(\mathbf{u}_{\varepsilon}+\mathbf{u}_{\mathbf{D}}\left(t_{\varepsilon}\right)\right): \tilde{e}\left(\mathbf{u}_{\varepsilon}+\mathbf{u}_{\mathbf{D}}\left(t_{\varepsilon}\right)\right) \mathrm{d} x \\
& \geq \int_{\Omega_{1} \cup \Omega_{2}} \mathbb{C}^{0} \tilde{e}\left(\mathbf{u}+\mathbf{u}_{\mathbf{D}}(t)\right): \tilde{e}\left(\mathbf{u}+\mathbf{u}_{\mathbf{D}}(t)\right) \mathrm{d} x,
\end{aligned}
$$

which concludes the proof of (3.21a).

Concerning the mutual recovery sequence, we first of all observe that inequality (3.21b) is nontrivial only when the right hand side is finite. In particular this implies that $\check{u} \in \mathcal{U}, \check{z} \in \mathcal{Z}$ and $z \geq \check{z} \geq 0$ on $\Gamma_{\mathrm{C}}$. As done in [21], merging ideas from [7] and [61], we define

$$
\begin{aligned}
& \left(\check{\mathrm{u}}_{\varepsilon}\right)_{\alpha}:=\check{\mathrm{u}}_{\alpha}, \quad \alpha=1,2, \\
& \left(\check{\mathrm{u}}_{\varepsilon}\right)_{3}:=\check{\mathrm{u}}_{3}+\varepsilon^{2} \eta_{\varepsilon}, \\
& \check{z}_{\varepsilon}:=\left\{\begin{array}{cc}
z_{\varepsilon} \check{z} / z & \text { if } z>0, \\
0 & \text { if } z=0,
\end{array}\right.
\end{aligned}
$$

with $\eta_{\varepsilon}\left(x_{1}, x_{2}, x_{3}\right):=\int_{0}^{x_{3}} \psi_{\varepsilon}\left(x_{1}, x_{2}, s\right) \mathrm{d} s$ as $x_{3} \in\left[-h_{1}, h_{2}\right]$ for some $\psi_{\varepsilon} \in C_{0}^{\infty}(\Omega)$ such that $\psi_{\varepsilon} \rightarrow \psi:=$ $-\sum_{\alpha, \beta=1}^{2} \mathbb{C}_{33 \alpha \beta} \tilde{e}\left(\check{\mathbf{u}}+\mathrm{u}_{\mathrm{D}}(t)\right)_{\alpha \beta} / \mathbb{C}_{3333}$ and $\varepsilon \partial \psi_{\varepsilon} / \partial x_{\alpha} \rightarrow 0$ in $L^{2}(\Omega)$ for $\alpha=1,2$.

It is easy to check that with this choice we have that

$$
\lim _{\varepsilon \rightarrow 0} \mathrm{R}_{\varepsilon}^{d}\left(\check{z}_{\varepsilon}-z_{\varepsilon}\right)=\mathrm{R}_{0}^{d}(\check{z}-z) .
$$

Thus, to prove the claim it suffices to deal with the convergence of the energy functional and the proof proceeds exactly as in [21, Lemma 4.5], with the only difference concerning the convergence of the term

$$
\int_{\Gamma_{\mathrm{C}}} \check{z}_{\varepsilon} \mathrm{Q}^{\varepsilon}\left(\llbracket \check{\mathrm{u}}_{\varepsilon} \rrbracket\right) \mathrm{d} \mathscr{H}^{2}
$$

which we are going to discuss here. Under the non restrictive assumption $\mathrm{E}_{0, \kappa}(t, \check{\mathrm{u}}, \check{z})<+\infty$, which implies

we have that the sequence

$$
\check{z} \llbracket \check{u} \rrbracket_{\sigma}=0 \quad \text { if } q_{\sigma}<0, \quad \sigma=\tau, \nu,
$$

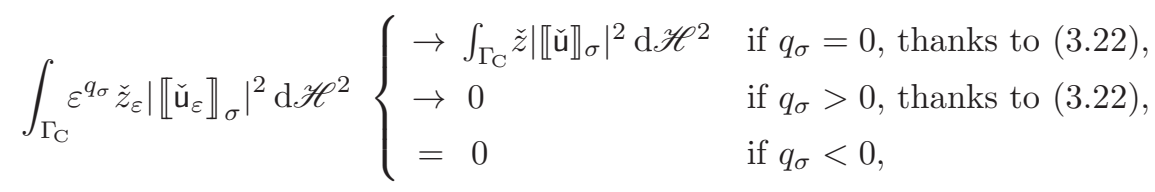

where the last line is because in that case $\check{z}_{\varepsilon}\left|\llbracket \check{u}_{\varepsilon} \rrbracket_{\sigma}\right|^{2}=\check{z}_{\varepsilon}\left|\llbracket \check{u} \rrbracket_{\sigma}\right|^{2}=0$ by (3.26) and by definition of $\check{z}_{\varepsilon}$. Summarizing we have therefore

$$
\int_{\Gamma_{\mathrm{C}}} \check{z}_{\varepsilon} \mathrm{Q}^{\varepsilon}\left(\llbracket \check{\mathrm{u}}_{\varepsilon} \rrbracket\right) \mathrm{d} \mathscr{H}^{2} \rightarrow \int_{\Gamma_{\mathrm{C}}} \check{z} \mathrm{Q}^{0}(\llbracket \check{\mathrm{u}} \rrbracket) \mathrm{d} \mathscr{H}^{2},
$$

which concludes the proof. 
Let us remark that the mutual recovery sequence condition stated in [50] is

$$
\limsup _{\varepsilon \rightarrow 0}\left[\mathrm{E}_{\varepsilon}\left(t_{\varepsilon}, \check{\mathrm{u}}_{\varepsilon}, \check{z}_{\varepsilon}\right)+\mathrm{R}_{\varepsilon}^{d}\left(\check{z}_{\varepsilon}-z_{\varepsilon}\right)-\mathrm{E}_{\varepsilon}\left(t_{\varepsilon}, \mathrm{u}_{\varepsilon}, z_{\varepsilon}\right)\right] \leq \mathrm{E}_{0}(t, \check{\mathrm{u}}, \check{z})+\mathrm{R}_{0}^{d}(\check{z}-z)-\mathrm{E}_{0}(t, \mathrm{u}, z)
$$

where $\left(\mathrm{u}_{\varepsilon}, z_{\varepsilon}\right)$ is a stable sequence converging to $(\mathrm{u}, z)$. This is, in general, slightly weaker than $(3.21 \mathrm{~b})$, as proven in [50, Prop.2.2]. Anyhow, here proving right (3.21b) was even simpler.

The dimension reduction result is summarized in the next statement which can be proven by following the same lines of [21, Theorem 5.5].

Theorem 3.2 (Convergence for $\varepsilon \rightarrow 0)$. Let $\mathrm{q}_{0}=\left(\mathrm{u}_{0}, z_{0}\right) \in \mathcal{Q}$ and, for any $\varepsilon>0$, let $\mathrm{q}_{0, \varepsilon}=\left(\mathrm{u}_{0, \varepsilon}, z_{0, \varepsilon}\right) \in$ $\mathcal{S}_{\varepsilon}(0)$ with $\mathcal{S}_{\varepsilon}$ as in $(1.5)$ with $\mathrm{E}_{\varepsilon}$ and $\mathrm{R}_{0}^{d}$ in place of $\mathcal{E}_{\varepsilon}$ and $\mathcal{R}_{\varepsilon}$, and let $\mathrm{q}_{0, \varepsilon} \stackrel{*}{\rightarrow} \mathrm{q}_{0}$ and $\mathrm{E}_{\varepsilon}\left(0, \mathrm{q}_{0, \varepsilon}\right) \rightarrow \mathrm{E}_{0}\left(0, \mathrm{q}_{0}\right)$ as $\varepsilon \rightarrow 0$. Let further $\mathrm{q}_{\varepsilon}=\left(\mathrm{u}_{\varepsilon}, z_{\varepsilon}\right):[0, T] \rightarrow \mathcal{Q}$ be an energetic solution to the problem $\left(\mathcal{U} \times \mathcal{Z}, \mathrm{E}_{\varepsilon}, \mathrm{R}_{\varepsilon}^{d}, \mathrm{q}_{0, \varepsilon}\right)$. Then, there exist $\mathrm{q}=(\mathrm{u}, z):[0, T] \rightarrow \mathcal{Q}$ and a subsequence (indexed, for simplicity, again by $\varepsilon$ ) such that

$$
\begin{aligned}
& \mathrm{E}_{\varepsilon}\left(t, \mathrm{q}_{\varepsilon}(t)\right) \rightarrow \mathrm{E}_{0}(t, \mathrm{q}(t)) \\
& \mathrm{R}_{\varepsilon}^{d}\left(z_{\varepsilon}(t)-z_{\varepsilon}(0)\right) \rightarrow \mathrm{R}_{0}^{d}(z(t)-z(0)) \\
& \partial_{t} \mathrm{E}_{\varepsilon}\left(\cdot, \mathrm{q}_{\varepsilon}(\cdot)\right) \rightarrow \partial_{t} \mathrm{E}_{0}(\cdot, \mathrm{q}(\cdot)) \\
& z_{\varepsilon}(t) \stackrel{*}{\rightarrow} z(t) \\
& \mathrm{u}_{\varepsilon}(t) \rightarrow \mathrm{u}(t)
\end{aligned}
$$

$$
\begin{aligned}
& \text { for every } t \in[0, T], \\
& \text { for every } t \in[0, T], \\
& \text { in } L^{1}(0, T) \text {, } \\
& \text { in } L^{\infty}\left(\Gamma_{\mathrm{C}}\right) \text { for every } t \in[0, T], \\
& \text { in } W^{1,2}\left(\Omega_{1} \cup \Omega_{2} ; \mathbb{R}^{3}\right) \text { for every } t \in[0, T] \text {. }
\end{aligned}
$$

Moreover, any $\mathrm{q}$ obtained in this way is an energetic solution to the problem $\left(\mathcal{U} \times \mathcal{Z}, \mathrm{E}_{0}, \mathrm{R}_{0}^{d}, \mathrm{q}_{0}\right)$.

The proof, as already mentioned, follows the same lines of [21, Theorem 5.5] and therefore is omitted. However, the reader can get an insight of this proof by looking at Section 5 where the proof of a more complicated case is provided.

Remark 3.3 (Isotropic material). The most typical case of $\mathbb{C}$ is $\mathbb{C}_{i j k l}=\lambda \delta_{i j} \delta_{k l}+\mu\left(\delta_{i k} \delta_{j l}+\delta_{i l} \delta_{j k}\right)$ with $\mu>0$ the so-called shear modulus, $\lambda$ is the so-called bulk modulus, $\lambda+\frac{2}{3} \mu>0$. Then the corresponding quadratic form becomes $\frac{1}{2} \mathbb{C} e: e=\mu|e|^{2}+\frac{1}{2} \lambda|\operatorname{tr} e|^{2}$ and the quadratic form (3.14) determining $\mathbb{C}^{0}$ from (3.13) is $\frac{1}{2} \mathbb{C}^{0} \tilde{e}: \tilde{e}=2 \mu \tilde{e}_{12}^{2}+\mu\left(\tilde{e}_{11}^{2}+\tilde{e}_{22}^{2}\right)+\frac{\mu \lambda}{2 \mu+\lambda}\left(\tilde{e}_{11}+\tilde{e}_{22}\right)^{2}$, cf. [7].

Remark 3.4 (Scaling of the adhesive response). The choice of the quadratic term $\mathrm{Q}^{\varepsilon}$ in (3.7) allows for an anisotropic behavior of the adhesive. In fact, the three-dimensional plate which is the starting point of our asymptotic analysis corresponds to the choice $\varepsilon=1$. This means that the elastic response of the adhesive in the 3D model is characterized by the quadratic form

$$
\mathrm{Q}^{1}(\llbracket \mathrm{u} \rrbracket):=\kappa_{\tau}\left|\llbracket \mathrm{u} \rrbracket_{\tau}\right|^{2}+\kappa_{\nu}\left|\llbracket \mathrm{u} \rrbracket_{\nu}\right|^{2}
$$

and the case $\kappa_{\tau}=\kappa_{\nu}$ corresponds to an isotropic behavior of the adhesive. In the limit model, the response of the adhesive is described by the quadratic form $\mathrm{Q}^{0}$ defined in (3.15). As already remarked, the choice $\kappa_{\tau}=\kappa_{\nu}=\kappa$ and $q_{\tau}=q_{\nu}=0$ (which corresponds to an isotropic scaling) gives $\mathrm{Q}^{0}(\llbracket \mathrm{u} \rrbracket)=\kappa|\llbracket \mathrm{u} \rrbracket|^{2}$. If $q_{\nu}>0$ and $q_{\tau}>0$, the term involving $\mathrm{Q}^{\varepsilon}$ is so small that it simply disappears and thus the limit problem describes the non-adhesive Signorini contact. In particular, $z$ will not evolve at all. The too-early-jump effect which energetic solutions may produce in non-convex problems trivially disappears in the limit. If instead $q_{\nu}<0$ and $q_{\tau}<0$ then the limit turns out to be a brittle delamination problem while, on the other hand, if $q_{\nu}=q_{\tau}=0$ then the limit problem describes a kind of adhesive contact. In the remaining cases we end up with a mix of brittle delamination and adhesive or sliding (non-adhesive) contact problems. Generally speaking, the choice of the scaling of the various terms in the expression of $\mathrm{Q}^{\varepsilon}$ has, to some extent, a purely mathematical meaning: indeed, different scalings simply allow us to capture different properties of the solutions of the 3D problem, cf. [57]. Nevertheless, in our case this choice can also be driven by the physics of the problem, namely, by the kind of approximation that we decide to pursue and that we want to incorporate in the limit problem. 
For constitutive reasons the elastic constants of the adhesive must satisfy the inequality $\kappa_{\nu} / \kappa_{\tau} \geq 2$, see Távara et al. [63]. If we know that $\kappa_{\tau}$ is much smaller with respect to $\kappa_{\nu}$, then we can decide to "simplify" the problem with essentially three kinds of approximations. In the first, $\kappa_{\tau}$ is so small that we choose to approximate the behavior of the adhesive with a Signorini contact problem in the plane and by an adhesive contact in the normal direction: this corresponds to the choice $q_{\tau}>0$ and $q_{\nu}=0$. In the second, $\kappa_{\nu}$ is so large that we choose the approximation with a brittle delamination problem in the normal direction and an adhesive contact in the plane: this corresponds to $q_{\tau}=0$ and $q_{\nu}<0$. In the third, $\kappa_{\tau}$ is so small and $\kappa_{\nu}$ so large that we choose to approximate with a Signorini contact in the plane and a brittle delamination in the normal: this corresponds to $q_{\tau}>0$ and $q_{\nu}<0$. Finally, we could instead take $q_{\tau}=q_{\nu}=0$ and obtain an adhesive problem with constants $\kappa_{\tau}$ and $\kappa_{\nu}$ also in the limit problem, without any approximation.

\section{Delaminating Kirchhoff-Love plate reformulated on $\Gamma_{C}$}

The aim of this section is to provide a truly two-dimensional formulation of the limit problem merely on $\Gamma_{\mathrm{C}}$.

Let us recall that $u \in W_{\mathrm{KL}}^{1,2}\left(\Omega_{1} \cup \Omega_{2} ; \mathbb{R}^{3}\right)$ if and only if there exist $\rho^{\ell}=\left(\rho_{1}^{\ell}, \rho_{2}^{\ell}\right) \in W^{1,2}\left(\Gamma_{\mathrm{C}} ; \mathbb{R}^{2}\right)$ and $\xi^{\ell} \in W^{2,2}\left(\Gamma_{\mathrm{C}}\right)$ such that

$$
\begin{aligned}
& \mathrm{u}_{\alpha}\left(x_{1}, x_{2}, x_{3}\right)=\left\{\begin{array}{ll}
\rho_{\alpha}^{1}\left(x_{1}, x_{2}\right)-x_{3} \frac{\partial}{\partial x_{\alpha}} \xi^{1}\left(x_{1}, x_{2}\right) & \text { if } x_{3} \in\left(-h_{1} / 2,0\right), \\
\rho_{\alpha}^{2}\left(x_{1}, x_{2}\right)-x_{3} \frac{\partial}{\partial x_{\alpha}} \xi^{2}\left(x_{1}, x_{2}\right) & \text { if } x_{3} \in\left(0, h_{2} / 2\right),
\end{array} \quad \text { with } \alpha=1,2,\right. \\
& \mathrm{u}_{3}\left(x_{1}, x_{2}, x_{3}\right)=\left\{\begin{array}{ll}
\xi^{1}\left(x_{1}, x_{2}\right) & \text { if } x_{3} \in\left(-h_{1} / 2,0\right), \\
\xi^{2}\left(x_{1}, x_{2}\right) & \text { if } x_{3} \in\left(0, h_{2} / 2\right),
\end{array} \text { for }\left(x_{1}, x_{2}\right) \in \Gamma_{\mathrm{C}},\right.
\end{aligned}
$$

see Le Dret [38, Lemma 4.2] for the case of a single plate. Since the effective domain of the limit energy $\mathrm{E}_{0, \kappa}$ is contained in the set of Kirchhoff-Love displacements $W_{\mathrm{KL}}^{1,2}\left(\Omega_{1} \cup \Omega_{2} ; \mathbb{R}^{3}\right)$ (see (3.17)), it is thus possible to rewrite the limit energy functionals in terms of the Kirchhoff-Love generalized displacements $\rho^{\ell}$ and $\xi^{\ell}$, with $\ell=1,2$.

Recalling the expression (3.4) of $\mathrm{u}_{\mathrm{D}}$, we first observe that

$$
\tilde{e}(\mathbf{u})=\left\{\begin{array}{ll}
\tilde{e}\left(\rho^{1}\right)-x_{3} \nabla^{2} \xi^{1} & \text { if } x_{3} \in\left(-h_{1} / 2,0\right), \\
\tilde{e}\left(\rho^{2}\right)-x_{3} \nabla^{2} \xi^{2} & \text { if } x_{3} \in\left(0, h_{2} / 2\right),
\end{array} \quad \text { and } \quad \tilde{e}\left(\mathbf{u}_{\mathrm{D}}\right)=\tilde{e}\left(\rho_{\mathrm{D}}\right)-x_{3} \nabla^{2} \xi_{\mathrm{D}}\right.
$$

for some $\rho_{\mathrm{D}}$ and $\xi_{\mathrm{D}}$ given. In terms of these new variables, we have

$$
\begin{aligned}
\frac{1}{2} \int_{\Omega_{1} \cup \Omega_{2}} \mathbb{C}^{0} \tilde{e}\left(\mathbf{u}+\mathbf{u}_{\mathrm{D}}\right): \tilde{e}\left(\mathbf{u}+\mathbf{u}_{\mathrm{D}}\right) \mathrm{d} x=\frac{1}{2} \sum_{\ell=1,2} \int_{\Gamma_{\mathrm{C}}} \frac{h_{\ell}}{2} \mathbb{C}^{0} \tilde{e}\left(\rho^{\ell}+\rho_{\mathrm{D}}\right): \tilde{e}\left(\rho^{\ell}+\rho_{\mathrm{D}}\right)+ \\
+(-1)^{\ell+1} \frac{h_{\ell}^{2}}{8} \mathbb{C}^{0} \tilde{e}\left(\rho^{\ell}+\rho_{\mathrm{D}}\right): \nabla^{2}\left(\xi^{\ell}+\xi_{\mathrm{D}}\right)+\frac{h_{\ell}^{3}}{24} \mathbb{C}^{0} \nabla^{2}\left(\xi^{\ell}+\xi_{\mathrm{D}}\right): \nabla^{2}\left(\xi^{\ell}+\xi_{\mathrm{D}}\right) \mathrm{d} \mathscr{H}^{2}
\end{aligned}
$$

while

$$
\begin{aligned}
\int_{\Omega_{1} \cup \Omega_{2}} \mathrm{f} \cdot\left(\mathrm{u}+\mathrm{u}_{\mathrm{D}}\right) \mathrm{d} x= & \sum_{\ell=1,2} \int_{\Gamma_{\mathrm{C}}} \varphi_{3}^{0}\left(\xi^{\ell}+\xi_{\mathrm{D}}\right)+\sum_{\alpha=1,2} \varphi_{\alpha}^{0}\left(\rho_{\alpha}^{\ell}+\left[\rho_{\mathrm{D}}\right]_{\alpha}\right)-\varphi_{\alpha}^{1} \frac{\partial}{\partial x_{\alpha}}\left(\xi^{\ell}+\xi_{\mathrm{D}}\right) \mathrm{d} \mathscr{H}^{2} \\
\text { with } \quad & \varphi_{\alpha}^{s}\left(x_{1}, x_{2}\right):=1_{0}\left(\ell_{\tau}\right) \int_{-h_{1} / 2}^{h_{2} / 2} x_{3}^{s} \mathrm{f}_{\alpha}\left(x_{1}, x_{2}, x_{3}\right) \mathrm{d} x_{3}, \quad\left(x_{1}, x_{2}\right) \in \Gamma_{\mathrm{C}} \\
& \varphi_{3}^{s}\left(x_{1}, x_{2}\right):=1_{0}\left(\ell_{\nu}\right) \int_{-h_{1} / 2}^{h_{2} / 2} x_{3}^{s} \mathrm{f}_{3}\left(x_{1}, x_{2}, x_{3}\right) \mathrm{d} x_{3}, \quad\left(x_{1}, x_{2}\right) \in \Gamma_{\mathrm{C}}
\end{aligned}
$$

where, for simplicity, we have not stressed the dependence of $\mathrm{u}_{\mathrm{D}}, \mathrm{f}, \rho_{\mathrm{D}}, \xi_{\mathrm{D}}$, and $\varphi_{i}^{s}$ on $t$. Since

$$
\left|\llbracket \mathrm{u} \rrbracket_{\tau}\right|^{2}=\sum_{\alpha=1}^{2}\left|\llbracket \mathrm{u}_{\alpha} \rrbracket\right|^{2}=\sum_{\alpha=1}^{2}\left|\rho_{\alpha}^{1}-\rho_{\alpha}^{2}\right|^{2}=\left|\rho^{1}-\rho^{2}\right|^{2}, \quad\left|\llbracket \mathrm{u} \rrbracket_{\nu}\right|^{2}=\left|\llbracket \mathrm{u}_{3} \rrbracket\right|^{2}=\left|\xi^{1}-\xi^{2}\right|^{2}
$$


we have that, according to (3.15),

$$
\mathrm{Q}^{0}(\llbracket \mathrm{u} \rrbracket)=1_{0}\left(q_{\tau}\right) \kappa_{\tau}\left|\rho^{1}-\rho^{2}\right|^{2}+1_{0}\left(q_{\nu}\right) \kappa_{\nu}\left|\xi^{1}-\xi^{2}\right|^{2} .
$$

In terms of the new variables, the set $A_{\mathrm{KL}}^{\text {ad }}$ becomes

$$
\begin{aligned}
\widehat{\mathrm{A}}_{\mathrm{KL}}^{\mathrm{ad}}:=\{ & (\rho, \xi, z) \in W^{1,2}\left(\Gamma_{\mathrm{C}} ; \mathbb{R}^{2}\right)^{2} \times W^{2,2}\left(\Gamma_{\mathrm{C}}\right)^{2} \times L^{\infty}\left(\Gamma_{\mathrm{C}}\right): 0 \leq z \leq 1, \xi^{1} \geq \xi^{2} \text { a.e. on } \Gamma_{\mathrm{C}}, \\
& z\left|\rho^{1}-\rho^{2}\right|=0 \text { if } q_{\tau}<0, z\left(\xi^{1}-\xi^{2}\right)=0 \text { if } q_{\nu}<0, \\
& \left.\left.\rho^{\ell}\right|_{\gamma_{\mathrm{D} \ell}}=0,\left.\quad \xi^{\ell}\right|_{\gamma_{\mathrm{D} \ell}}=\left.\nabla \xi^{\ell}\right|_{\gamma_{\mathrm{D} \ell}} \cdot \nu=0 \text { a.e. on } \gamma_{\mathrm{D} \ell}, \quad \ell=1,2\right\},
\end{aligned}
$$

and the stored energy is

$$
\widehat{\mathrm{E}}_{0}(t, \rho, \xi, z):=\left\{\begin{array}{cc}
\frac{1}{2} \int_{\Gamma_{\mathrm{C}}} \sum_{\ell=1,2}\left(\frac{h_{\ell}}{2} \mathbb{C}^{0} \tilde{e}\left(\rho^{\ell}+\rho_{\mathrm{D}}\right): \tilde{e}\left(\rho^{\ell}+\rho_{\mathrm{D}}\right)+(-1)^{\ell+1} \frac{h_{\ell}^{2}}{8} \mathbb{C}^{0} \tilde{e}\left(\rho^{\ell}+\rho_{\mathrm{D}}\right): \nabla^{2}\left(\xi^{\ell}+\xi_{\mathrm{D}}\right)\right. \\
\left.+\frac{h_{\ell}^{3}}{24} \mathbb{C}^{0} \nabla^{2}\left(\xi^{\ell}+\xi_{\mathrm{D}}\right): \nabla^{2}\left(\xi^{\ell}+\xi_{\mathrm{D}}\right)-\varphi_{3}^{0}\left(\xi^{\ell}+\xi_{\mathrm{D}}\right)-\varphi_{\alpha}^{0}\left(\rho_{\alpha}^{\ell}+\left[\rho_{\mathrm{D}}\right]_{\alpha}\right)+\varphi_{\alpha}^{1} \frac{\partial}{\partial x_{\alpha}}\left(\xi^{\ell}+\xi_{\mathrm{D}}\right)\right) \\
+z 1_{0}\left(q_{\tau}\right) \kappa_{\tau}\left|\rho^{1}-\rho^{2}\right|^{2}+z 1_{0}\left(q_{\nu}\right) \kappa_{\nu}\left|\xi^{1}-\xi^{2}\right|^{2} \mathrm{~d} \mathscr{H}^{2} & \text { if }(\rho, \xi, z) \in \widehat{\mathrm{A}}_{\mathrm{KL}}^{\mathrm{ad}}, \\
+\infty & \text { else. }
\end{array}\right.
$$

Moreover, we consider the dissipation $\mathrm{R}_{0}^{d}$ from (3.19). The limit problem admits therefore a purely $2 \mathrm{D}$ formulation, thanks to (4.1).

\section{Mode-sensitive delamination}

Engineering models of delamination are usually more complicated than presented here so far: the dissipation in the so-called mode I (delamination by opening) is less than in the so-called mode II (delamination by shearing); sometimes, the difference may be tens or even hundreds of percents, cf., e.g., [42, 45]. Recently, a quasistatic associative model has been devised, analyzed, and tested computationally in $[59,60]$. The purpose of this last section is to adapt the previously exposed asymptotic analysis to this modesensitive model.

\subsection{Formulation of the problem and scaling}

Microscopically, the additional dissipation in Mode II may be explained by a certain plastic process both in the adhesive itself and in a narrow bulk vicinity of the delamination surface before the actual delamination starts, cf. $[42,64]$. Following $[59,60]$, we are therefore led to consider an additional plastic-like inelastic process on $\Gamma_{\mathrm{C}}$ described by a further dissipative variable $\pi$ having the meaning of the "plastic" interfacial slip on $\Gamma_{\mathrm{C}}$. Denoting the delamination parameter by $\zeta$ in this section, the "rate-independent variable" $z$ then will have two components, i.e., $z=(\zeta, \pi)$.

Instead of $\widetilde{\mathcal{E}}_{\varepsilon}$ and $\mathcal{R}_{\varepsilon}$ from (2.1) and (2.3), we now consider the following energy functionals proposed in $[59,60]$

$$
\widetilde{\mathcal{E}}_{\varepsilon}(t, u, \zeta, \pi)= \begin{cases}\frac{1}{2} \int_{\Omega_{1}^{\varepsilon} \cup \Omega_{2}^{\varepsilon}} \operatorname{Ce}(u): e(u)-2 f^{\varepsilon}(t) \cdot u \mathrm{~d} x & \\ \quad+\int_{\Gamma_{\mathrm{C}}} \zeta Q^{\varepsilon}(\llbracket u \rrbracket-\mathbb{T} \pi)+\frac{\kappa_{\mathrm{H}}^{\varepsilon}}{2}|\pi|^{2}+\frac{\kappa_{0}^{\varepsilon}}{2}\left|\nabla_{\mathrm{S}} \pi\right|^{2} \mathrm{~d} \mathscr{H}^{2} & \text { if }(u, \zeta) \in \mathcal{A}_{\varepsilon}^{\text {ad }}\left(w_{\mathrm{D}}^{\varepsilon}(t)\right), \\ & \text { and } \pi \in W^{1,2}\left(\Gamma_{\mathrm{C}} ; \mathbb{R}^{2}\right), \\ +\infty & \text { else },\end{cases}
$$


where $\nabla_{\mathrm{S}}$ is the gradient along the two-dimensional surface $\Gamma_{\mathrm{C}}$ and $\mathbb{T}: \mathbb{R}^{2} \rightarrow \mathbb{R}^{3}:\left(\pi_{1}, \pi_{2}\right) \mapsto\left(\pi_{1}, \pi_{2}, 0\right)$, and

$$
\mathcal{R}_{\varepsilon}(\dot{\zeta}, \dot{\pi}):= \begin{cases}\int_{\Gamma_{\mathrm{C}}} a_{\varepsilon}|\dot{\zeta}|+\sigma_{\varepsilon, \text { yield }}|\dot{\pi}| \mathrm{d} \mathscr{H}^{2} & \text { if } \dot{\zeta} \leq 0 \text { on } \Gamma_{\mathrm{C}} \\ +\infty & \text { else. }\end{cases}
$$

Instead of $z$ from $(2.10), z=(\zeta, \pi)$ lives in the space

$$
z:=\left\{(\zeta, \pi) \in L^{\infty}\left(\Gamma_{\mathrm{C}}\right) \times W^{1,2}\left(\Gamma_{\mathrm{C}} ; \mathbb{R}^{2}\right) ; 0 \leq \zeta \leq 1 \text { on } \Gamma_{\mathrm{C}}\right\}
$$

The coefficient $\kappa_{\mathrm{H}}^{\varepsilon}>0$ is related with a kinematic-type hardening. The idea of this model is that under increasing load, in Mode II, first the interfacial slip starts evolving when the stress achieves the threshold $\sigma_{\varepsilon, \text { yield }}$ and, due to the hardening, the stress still continues growing until the threshold $a_{\varepsilon}$ activates the delamination $\zeta$ which then eventually stops also the evolution of $\pi$. This implies that the driving stress for $\pi$ has, after delamination, a magnitude less than $\sigma_{\varepsilon, \text { yield }}$. This scenario needs the condition

$$
\frac{1}{2} \kappa_{\tau}^{\varepsilon} a_{\varepsilon}<\sigma_{\varepsilon, \text { yield }}^{2} \leq 2 \kappa_{\tau}^{\varepsilon} a_{\varepsilon} \quad \text { with } \quad \kappa_{\tau}^{\varepsilon}=\left[Q^{\varepsilon}\right]_{\tau}^{\prime \prime}
$$

to be satisfied, cf. $\left[59\right.$, Formula (13.69)] or $\left[60\right.$, Formula (14)], where $\left[Q^{\varepsilon}\right]_{\tau}^{\prime \prime}$ here denotes the second tangential derivative of $Q^{\varepsilon}$. The validity of this model has been tested numerically in [60]. Dealing now for simplicity with the case $q_{\tau}=q_{\nu}=0$ in the definition (3.6) of $Q^{\varepsilon}$, from the relation above we obtain that

$$
\kappa_{\tau}^{\varepsilon}=2 \varepsilon k_{\tau} .
$$

Taking into account that $a_{\varepsilon}=\varepsilon^{3}$ a, cf. (3.10), the two-sided condition (5.2) turns into $\varepsilon^{4} \kappa_{\tau}$ a $\leq \sigma_{\varepsilon, \text { yield }}^{2} \leq$ $4 \varepsilon^{4} \kappa_{\tau}$ a. It leads us to set

$$
\sigma_{\varepsilon, \text { yield }}=\varepsilon^{2} \sigma_{\text {yield }}
$$

for some $\sigma_{\text {yield }}$ satisfying

$$
k_{\tau} \mathrm{a}<\sigma_{\text {yield }}^{2} \leq 4 k_{\tau} \text { a }
$$

The energy dissipated in Mode II, let us denote it by $a_{\varepsilon}^{\mathrm{II}}$, is

$$
a_{\varepsilon}^{\mathrm{II}}=a_{\varepsilon}+\frac{\sigma_{\varepsilon, \text { yield }} \sqrt{2 \kappa_{\mathrm{t}}^{\varepsilon} a_{\varepsilon}}-\sigma_{\varepsilon, \text { yield }}^{2}}{\kappa_{\mathrm{H}}^{\varepsilon}},
$$

cf. again $[59,60]$, and then the so-called fracture-mode sensitivity $a_{\varepsilon}^{\mathrm{II}} / a_{\varepsilon}$ is indeed bigger than 1 as expected in engineering models, namely

$$
\frac{a_{\varepsilon}^{\mathrm{II}}}{a_{\varepsilon}}=1+\frac{\sigma_{\varepsilon, \mathrm{yield}}}{\kappa_{\mathrm{H}}^{\varepsilon}} \sqrt{\frac{2 \kappa_{\mathrm{t}}^{\varepsilon}}{a_{\varepsilon}}}-\frac{\sigma_{\varepsilon, \mathrm{yield}}^{2}}{\kappa_{\mathrm{H}}^{\varepsilon} a_{\varepsilon}} .
$$

Setting

$$
\kappa_{\mathrm{H}}^{\varepsilon}=\varepsilon \kappa_{\mathrm{H}}, \quad \kappa_{\mathrm{H}}>0,
$$

we can control $a_{\varepsilon}^{\mathrm{II}} / a_{\varepsilon}$ uniformly with respect to $\varepsilon$.

Let us remark that, to fit it more straightforwardly to the previous sections, we used the model with $\nabla_{\mathrm{S}} \pi$, as suggested in [60, Remark 3]. Then this model is indeed an extension of the previous model in the sense that, if $\sigma_{\text {yield }}>4 k_{\tau}$ a and $\pi(0)=0$, no plastic slip is ever triggered and this model gives the same response as that one in Section 2. 
Following the same lines traced at the end of Section 2, instead of (5.1a), we can consider

$$
\mathcal{E}_{\varepsilon}(t, \mathrm{u}, \zeta, \pi)= \begin{cases}\frac{1}{2} \int_{\Omega_{1}^{\varepsilon} \cup \Omega_{2}^{\varepsilon}} \mathbb{C} e\left(u+u_{D}^{\varepsilon}(t)\right): e\left(u+u_{D}^{\varepsilon}(t)\right)-2 f^{\varepsilon}(t) \cdot\left(u+u_{D}^{\varepsilon}(t)\right) \mathrm{d} x & \\ \quad+\int_{\Gamma_{\mathrm{C}}} \zeta Q^{\varepsilon}(\llbracket u \rrbracket-\mathbb{T} \pi)+\frac{\kappa_{\mathrm{H}}^{\varepsilon}}{2}|\pi|^{2}+\frac{\kappa_{0}^{\varepsilon}}{2}\left|\nabla_{\mathrm{S}} \pi\right|^{2} \mathrm{~d} \mathscr{H}^{2} & \text { if }(u, \zeta) \in \mathcal{A}_{\varepsilon}^{\mathrm{ad}} \\ +\infty & \text { and } \pi \in W^{1,2}\left(\Gamma_{\mathrm{C}}, \mathbb{R}^{2}\right) \\ & \text { else. }\end{cases}
$$

Scaling displacements and body forces and choosing Dirichlet boundary conditions as at the beginning of Section 3.1, we introduce the scaled interfacial slip

$$
\pi:=\frac{1}{\varepsilon} \pi \circ p_{\varepsilon}
$$

and set

$$
\kappa_{0}^{\varepsilon}=\varepsilon \kappa_{0}, \quad \kappa_{0}>0
$$

in order to obtain

$$
\varepsilon_{\varepsilon}(t, u, \zeta, \pi)=\varepsilon^{3} \mathrm{E}_{\varepsilon}(t, \mathrm{u}, \zeta, \pi)
$$

for $(u, \zeta) \in \mathcal{A}_{\varepsilon}^{\mathrm{ad}}$, where

$$
\mathrm{E}_{\varepsilon}(t, \mathrm{u}, \zeta, \boldsymbol{\pi})= \begin{cases}\frac{1}{2} \int_{\Omega_{1} \cup \Omega_{2}} \mathrm{Ce}^{\varepsilon}\left(\mathrm{u}+\mathrm{u}_{\mathrm{D}}(t)\right): e^{\varepsilon}\left(\mathrm{u}+\mathrm{u}_{\mathrm{D}}(t)\right)-2 \mathrm{f}^{\varepsilon}(t) \cdot\left(\mathrm{u}+\mathrm{u}_{\mathrm{D}}(t)\right) \mathrm{d} x & \\ \quad+\int_{\Gamma_{\mathrm{C}}} \zeta \mathrm{Q}^{\varepsilon}(\llbracket \mathrm{u} \rrbracket-\mathbb{T} \boldsymbol{\pi})+\frac{\kappa_{\mathrm{H}}}{2}|\boldsymbol{\pi}|^{2}+\frac{\kappa_{0}}{2}\left|\nabla_{\mathrm{S}} \boldsymbol{\pi}\right|^{2} \mathrm{~d} \mathscr{H}^{2} & \text { if }(\mathrm{u}, \zeta) \in \mathrm{A}^{\text {ad }} \\ +\infty & \text { and } \boldsymbol{\pi} \in W^{1,2}\left(\Gamma_{\mathrm{C}}, \mathbb{R}^{2}\right) \\ & \text { else }\end{cases}
$$

Concerning the dissipation energy, contrarily to what we have done in Section 3, we consider here only the scaling that leads to a non trivial limit dissipation; namely, under the scalings (3.10) with $d=0$ and (5.3), we have

$$
\mathcal{R}_{\varepsilon}(\dot{\zeta}, \dot{\pi})=\varepsilon^{3} \mathrm{R}(\dot{\zeta}, \dot{\pi})
$$

where

$$
\mathrm{R}(\dot{\zeta}, \dot{\pi}):= \begin{cases}\int_{\Gamma_{\mathrm{C}}} \mathrm{a}|\dot{\zeta}|+\sigma_{\text {yield }}|\dot{\pi}| \mathrm{d} \mathscr{H}^{2} & \text { if } \dot{\zeta} \leq 0 \text { on } \Gamma_{\mathrm{C}} \\ +\infty & \text { else. }\end{cases}
$$

Then we define

$$
\mathrm{E}_{0}(t, \mathrm{u}, \zeta, \boldsymbol{\pi})= \begin{cases}\frac{1}{2} \int_{\Omega_{1} \cup \Omega_{2}} \mathbb{C}^{0} \tilde{e}\left(\mathrm{u}+\mathrm{u}_{\mathrm{D}}(t)\right): \tilde{e}\left(\mathrm{u}+\mathrm{u}_{\mathrm{D}}(t)\right)-2 \mathrm{f}^{0}(t) \cdot\left(\mathrm{u}+\mathrm{u}_{\mathrm{D}}(t)\right) \mathrm{d} x & \\ \quad+\int_{\Gamma_{\mathrm{C}}} \zeta \mathrm{Q}^{0}(\llbracket \mathrm{u} \rrbracket-\mathbb{T} \boldsymbol{\pi})+\frac{\kappa_{\mathrm{H}}}{2}|\boldsymbol{\pi}|^{2}+\frac{\kappa_{0}}{2}\left|\nabla_{\mathrm{S}} \boldsymbol{\pi}\right|^{2} \mathrm{~d} \mathscr{H}^{2} & \text { if }(\mathrm{u}, \zeta) \in \mathrm{A}_{\mathrm{KL}}^{\mathrm{ad}} \\ & \text { and } \boldsymbol{\pi} \in W^{1,2}\left(\Gamma_{\mathrm{C}}, \mathbb{R}^{2}\right) \\ +\infty & \text { else }\end{cases}
$$

where now

$$
\mathrm{A}_{\mathrm{KL}}^{\mathrm{ad}}=\left\{(\mathrm{u}, \zeta) \in \mathrm{A}^{\mathrm{ad}}: \mathrm{u} \in W_{\mathrm{KL}}^{1,2}\left(\Omega_{1} \cup \Omega_{2} ; \mathbb{R}^{3}\right)\right\}
$$




\subsection{Dimension reduction}

Now we are going to perform the same analysis (dimension reduction and relation between energetic solutions associated to the rescaled and the limit problems) that we discussed in Section 3, providing in particular the link between the functionals $E_{\varepsilon}$ and $E_{0}$. Again, the main idea is to apply the abstract scheme developed in [50]. This case involving mixed modes of delamination is more complicated and requires much detailed adaptations. Also for the sake of clarity, we preferred to give the whole proofs.

In the next lemma we prove a lower bound for the limit energies.

Lemma 5.1 (Lower semicontinuity). If $t_{\varepsilon} \rightarrow t, \zeta_{\varepsilon} \stackrel{*}{\rightarrow} \zeta$ in $L^{\infty}\left(\Gamma_{\mathrm{C}}\right), 0 \leq \zeta_{\varepsilon} \leq 1$ a.e., $\boldsymbol{\pi}_{\varepsilon} \rightarrow \boldsymbol{\pi}$ in $W^{1,2}\left(\Gamma_{\mathrm{C}} ; \mathbb{R}^{2}\right)$ and $\mathrm{u}_{\varepsilon} \rightarrow \mathrm{u}$ in $W^{1,2}\left(\Omega_{1} \cup \Omega_{2} ; \mathbb{R}^{3}\right)$ as $\varepsilon \rightarrow 0^{+}$, then

$$
\liminf _{\varepsilon \rightarrow 0^{+}} \mathrm{E}_{\varepsilon}\left(t_{\varepsilon}, \mathrm{u}_{\varepsilon}, \zeta_{\varepsilon}, \boldsymbol{\pi}_{\varepsilon}\right) \geq \mathrm{E}_{0}(t, \mathrm{u}, \zeta, \boldsymbol{\pi}) .
$$

Proof. Under the assumption that the liminf on the left-hand sides be finite, by the positive definiteness of $\mathbb{C}\left(\right.$ see $(2.4)$ ) and the continuity of $w$ with respect to $t$, it follows that, up to subsequences, $\left\|e^{\varepsilon}\left(u_{\varepsilon}\right)\right\|_{L^{2}} \leq C$ and hence $\left\|e\left(\mathbf{u}_{\varepsilon}\right)_{i 3}\right\|_{L^{2}} \leq C \varepsilon, i=1,2,3$; thus, passing to the limit, we obtain $e(\mathbf{u})_{i 3}=0$ which implies $\mathrm{u} \in W_{\mathrm{KL}}^{1,2}\left(\Omega_{1} \cup \Omega_{2} ; \mathbb{R}^{3}\right)$. By this fact and by Lemma 3.1 we have that also the right-hand side in $(5.9)$ is finite and that it suffices to prove that

$$
\begin{array}{r}
\liminf _{\varepsilon \rightarrow 0^{+}} \int_{\Omega_{1} \cup \Omega_{2}} \mathbb{C} e^{\varepsilon}\left(\mathrm{u}_{\varepsilon}+\mathrm{w}\left(t_{\varepsilon}\right)\right): e^{\varepsilon}\left(\mathrm{u}_{\varepsilon}+\mathrm{w}\left(t_{\varepsilon}\right)\right) \mathrm{d} x+\int_{\Gamma_{\mathrm{C}}} \zeta_{\varepsilon} \mathrm{Q}^{\varepsilon}\left(\llbracket \mathrm{u}_{\varepsilon} \rrbracket-\mathbb{T} \boldsymbol{\pi}_{\varepsilon}\right)+\frac{\kappa_{\mathrm{H}}}{2}\left|\boldsymbol{\pi}_{\varepsilon}\right|^{2}+\frac{\kappa_{0}}{2}\left|\nabla_{\mathrm{S}} \boldsymbol{\pi}_{\varepsilon}\right|^{2} \mathrm{~d} \mathscr{H}^{2} \geq \\
\geq \int_{\Omega_{1} \cup \Omega_{2}} \mathbb{C}^{0} \tilde{e}(\mathrm{u}+\mathrm{w}(t)): \tilde{e}(\mathrm{u}+\mathrm{w}(t)) \mathrm{d} x+\int_{\Gamma_{\mathrm{C}}} \zeta \mathrm{Q}^{0}(\llbracket \mathrm{u} \rrbracket-\mathbb{T} \boldsymbol{\pi})+\frac{\kappa_{\mathrm{H}}}{2}|\boldsymbol{\pi}|^{2}+\frac{\kappa_{0}}{2}\left|\nabla_{\mathrm{S}} \boldsymbol{\pi}\right|^{2} \mathrm{~d} \mathscr{H}^{2} .
\end{array}
$$

The bulk term can be treated as we did in the proof of (3.21a) noticing that, for $\alpha, \beta=1,2$,

$$
e^{\varepsilon}\left(\mathbf{u}_{\varepsilon}\right)_{\alpha \beta}=e\left(\mathbf{u}_{\varepsilon}\right)_{\alpha \beta} \rightarrow e(\mathbf{u})_{\alpha \beta} \quad \text { in } L^{2}\left(\Omega_{1} \cup \Omega_{2}\right),
$$

using property (3.14) and the continuity of $w$ with respect to $t$ to obtain

$$
\begin{aligned}
\liminf _{\varepsilon \rightarrow 0^{+}} \int_{\Omega_{1} \cup \Omega_{2}} \mathbb{C} e^{\varepsilon}\left(\mathbf{u}_{\varepsilon}+\mathbf{w}\left(t_{\varepsilon}\right)\right): e^{\varepsilon}\left(\mathbf{u}_{\varepsilon}+\mathbf{w}\left(t_{\varepsilon}\right)\right) \mathrm{d} x & \geq \liminf _{\varepsilon \rightarrow 0^{+}} \int_{\Omega_{1} \cup \Omega_{2}} \mathbb{C}^{0} \tilde{e}\left(\mathbf{u}_{\varepsilon}+\mathbf{w}\left(t_{\varepsilon}\right)\right): \tilde{e}\left(\mathbf{u}_{\varepsilon}+\mathbf{w}\left(t_{\varepsilon}\right)\right) \mathrm{d} x \\
& \geq \int_{\Omega_{1} \cup \Omega_{2}} \mathbb{C}^{0} \tilde{e}(\mathbf{u}+\mathbf{w}(t)): \tilde{e}(\mathbf{u}+\mathbf{w}(t)) \mathrm{d} x
\end{aligned}
$$

Since $\boldsymbol{\pi}_{\varepsilon} \rightarrow \boldsymbol{\pi}$ in $W^{1,2}\left(\Gamma_{\mathrm{C}} ; \mathbb{R}^{2}\right)$ then we immediately get

$$
\liminf _{\varepsilon \rightarrow 0^{+}} \int_{\Gamma_{\mathrm{C}}} \frac{\kappa_{\mathrm{H}}}{2}\left|\boldsymbol{\pi}_{\varepsilon}\right|^{2}+\frac{\kappa_{0}}{2}\left|\nabla_{\mathrm{S}} \boldsymbol{\pi}_{\varepsilon}\right|^{2} \mathrm{~d} \mathscr{H}^{2} \geq \int_{\Gamma_{\mathrm{C}}} \frac{\kappa_{\mathrm{H}}}{2}|\boldsymbol{\pi}|^{2}+\frac{\kappa_{0}}{2}\left|\nabla_{\mathrm{S}} \boldsymbol{\pi}\right|^{2} \mathrm{~d} \mathscr{H}^{2} .
$$

Finally, concerning the term involving $Q^{\varepsilon}$ we have

$$
\begin{aligned}
\lim _{\varepsilon \rightarrow 0^{+}} \int_{\Gamma_{\mathrm{C}}} \zeta_{\varepsilon} \mathrm{Q}^{\varepsilon}\left(\llbracket \mathrm{u}_{\varepsilon} \rrbracket-\mathbb{T} \boldsymbol{\pi}_{\varepsilon}\right) \mathrm{d} \mathscr{H}^{2} & =\lim _{\varepsilon \rightarrow 0^{+}} \int_{\Gamma_{\mathrm{C}}} \zeta_{\varepsilon}\left(\kappa_{\tau}\left|\llbracket \mathrm{u}_{\varepsilon} \rrbracket_{\tau}-\boldsymbol{\pi}_{\varepsilon}\right|^{2}+\kappa_{\nu}\left|\llbracket \mathrm{u}_{\varepsilon} \rrbracket_{\nu}\right|^{2}\right) \mathrm{d} \mathscr{H}^{2} \\
& =\int_{\Gamma_{\mathrm{C}}} \zeta\left(\kappa_{\tau}\left|\llbracket \mathrm{u} \rrbracket_{\tau}-\boldsymbol{\pi}\right|^{2}+\kappa_{\nu}\left|\llbracket \mathrm{u} \rrbracket_{\nu}\right|^{2}\right) \mathrm{d} \mathscr{H}^{2} \\
& =\int_{\Gamma_{\mathrm{C}}} \zeta \mathrm{Q}^{0}(\llbracket \mathrm{u} \rrbracket-\mathbb{T} \boldsymbol{\pi}) \mathrm{d} \mathscr{H}^{2}
\end{aligned}
$$

where in the first and last equalities we used the definitions of $Q^{\varepsilon}$ and $Q^{0}$ respectively, while the second follows by observing that, by our assumptions,

$$
\kappa_{\tau}\left|\llbracket \mathrm{u}_{\varepsilon} \rrbracket_{\tau}-\boldsymbol{\pi}_{\varepsilon}\right|^{2}+\kappa_{\nu}\left|\llbracket \mathrm{u}_{\varepsilon} \rrbracket_{\nu}\right|^{2} \rightarrow \kappa_{\tau}\left|\llbracket \mathrm{u} \rrbracket_{\tau}-\boldsymbol{\pi}\right|^{2}+\kappa_{\nu}\left|\llbracket \mathrm{u} \rrbracket_{\nu}\right|^{2} \text { in } L^{1}\left(\Gamma_{\mathrm{C}}\right),
$$

while $\zeta_{\varepsilon} \stackrel{*}{\rightarrow} \zeta$ in $L^{\infty}\left(\Gamma_{\mathrm{C}}\right)$. 
The next lemma is fundamental to apply the $\Gamma$-convergence scheme developed in [50].

Lemma 5.2 (Mutual recovery sequence). Let $t \in[0, T]$, and $(\mathrm{u},(\zeta, \pi))) \in \mathcal{U} \times \mathbf{z}$. For every uniformly stable sequence $\left(t_{\varepsilon}, \mathrm{u}_{\varepsilon},\left(\zeta_{\varepsilon}, \boldsymbol{\pi}_{\varepsilon}\right)\right) \in[0, T] \times \mathcal{U} \times z$ such that $t_{\varepsilon} \rightarrow t$ in $[0, T], \mathrm{u}_{\varepsilon} \rightarrow \mathrm{u}$ in $W^{1,2}\left(\Omega_{1} \cup \Omega_{2} ; \mathbb{R}^{3}\right)$, $\zeta_{\varepsilon} \stackrel{*}{\rightarrow} \zeta$ in $L^{\infty}\left(\Gamma_{\mathrm{C}}\right), \boldsymbol{\pi}_{\varepsilon} \rightarrow \boldsymbol{\pi}$ in $W^{1,2}\left(\Gamma_{\mathrm{C}} ; \mathbb{R}^{2}\right)$, and for every $(\check{\mathrm{u}},(\check{\zeta}, \check{\boldsymbol{\pi}})) \in \mathcal{U} \times \boldsymbol{z}$, there exist $\check{\mathbf{u}}_{\varepsilon} \in \mathcal{U}$ and $\left(\check{\zeta}_{\varepsilon}, \check{\pi}_{\varepsilon}\right) \in Z$ such that

$$
\begin{aligned}
& \check{\mathrm{u}}_{\varepsilon} \rightarrow \text { ú } \text { in } W^{1,2}\left(\Omega_{1} \cup \Omega_{2} ; \mathbb{R}^{3}\right), \\
& \check{\zeta}_{\varepsilon} \stackrel{*}{\rightarrow} \check{\zeta} \text { in } L^{\infty}\left(\Gamma_{\mathrm{C}}\right), \\
& \check{\boldsymbol{\pi}}_{\varepsilon} \rightarrow \check{\boldsymbol{\pi}} \text { in } W^{1,2}\left(\Gamma_{\mathrm{C}} ; \mathbb{R}^{2}\right), \\
& \limsup _{\varepsilon \rightarrow 0^{+}}\left[\mathrm{E}_{\varepsilon}\left(t_{\varepsilon}, \check{\mathrm{u}}_{\varepsilon}, \check{\zeta}_{\varepsilon}, \check{\boldsymbol{\pi}}_{\varepsilon}\right)+\mathrm{R}\left(\check{\zeta}_{\varepsilon}-\zeta_{\varepsilon}, \check{\boldsymbol{\pi}}_{\varepsilon}-\boldsymbol{\pi}_{\varepsilon}\right)-\mathrm{E}_{\varepsilon}\left(t_{\varepsilon}, \mathrm{u}_{\varepsilon}, \zeta_{\varepsilon}, \boldsymbol{\pi}_{\varepsilon}\right)\right] \\
& \quad \leq \mathrm{E}_{0}(t, \check{\mathrm{u}}, \check{\zeta}, \check{\boldsymbol{\pi}})+\mathrm{R}(\check{\zeta}-\zeta, \check{\boldsymbol{\pi}}-\boldsymbol{\pi})-\mathrm{E}_{0}(t, \mathrm{u}, \zeta, \boldsymbol{\pi}) .
\end{aligned}
$$

Moreover, setting for any $\varepsilon \geq 0$

$$
\overline{\mathrm{E}}_{\varepsilon}(t, \mathrm{u}, \zeta, \boldsymbol{\pi}):=\mathrm{E}_{\varepsilon}(t, \mathrm{u}, \zeta, \boldsymbol{\pi})-\int_{\Gamma_{\mathrm{C}}} \frac{\kappa_{0}}{2}\left|\nabla_{\mathrm{S}} \boldsymbol{\pi}\right|^{2} \mathrm{~d} \mathscr{H}^{2},
$$

then we have

$$
\limsup _{\varepsilon \rightarrow 0^{+}}\left[\overline{\mathrm{E}}_{\varepsilon}\left(t_{\varepsilon}, \check{\mathrm{u}}_{\varepsilon}, \check{\zeta}_{\varepsilon}, \check{\boldsymbol{\pi}}_{\varepsilon}\right)+\mathrm{R}\left(\check{\zeta}_{\varepsilon}-\zeta_{\varepsilon}, \check{\boldsymbol{\pi}}_{\varepsilon}-\boldsymbol{\pi}_{\varepsilon}\right)\right] \leq \overline{\mathrm{E}}_{0}(t, \check{\mathrm{u}}, \check{\zeta}, \check{\boldsymbol{\pi}})+\mathrm{R}(\check{\zeta}-\zeta, \check{\boldsymbol{\pi}}-\boldsymbol{\pi}) .
$$

Proof. First of all we notice that, since the sequence $\left(t_{\varepsilon}, \mathrm{u}_{\varepsilon},\left(\zeta_{\varepsilon}, \boldsymbol{\pi}_{\varepsilon}\right)\right)$ is uniformly stable, by the lower semicontinuity Lemma 5.1 we have that $\mathrm{E}_{0}(t, \mathrm{u}, \zeta, \pi)<+\infty$. Moreover we can assume that righthand side in (5.14) (and then in (5.16)) be finite, otherwise the result is achieved by simply taking $\left(\check{\mathrm{u}}_{\varepsilon},\left(\check{\zeta}_{\varepsilon}, \check{\boldsymbol{\pi}}_{\varepsilon}\right)\right)=(\check{\mathrm{u}},(\check{\zeta}, \check{\boldsymbol{\pi}}))$ for any $\varepsilon>0$. This non-resctrictive assumption, together with the finiteness of $\mathrm{E}_{0}(t, \mathrm{u}, \zeta, \boldsymbol{\pi})$, implies that

$$
\mathrm{E}_{0}(t, \check{\mathrm{u}}, \check{\zeta}, \check{\boldsymbol{\pi}})<+\infty \quad \text { and } \quad \mathrm{R}(\check{\zeta}-\zeta, \check{\pi}-\boldsymbol{\pi})<+\infty .
$$

In particular then we have $\zeta \geq \check{\zeta}$ a.e. on $\Gamma_{\mathrm{C}}$. Inspired by [60], let us define

$$
\check{\zeta}_{\varepsilon}:=\left\{\begin{array}{ll}
\frac{\zeta_{\varepsilon} \check{\zeta}}{\zeta} & \text { if } \zeta>0, \\
0 & \text { if } \zeta=0,
\end{array} \quad \text { and } \quad \check{\pi}_{\varepsilon}:=\check{\pi}+\pi_{\varepsilon}-\pi .\right.
$$

Then it is easy to check that $0 \leq \check{\zeta}_{\varepsilon} \leq 1$ a.e. on $\Gamma_{\mathrm{C}}, \check{\zeta}_{\varepsilon} \stackrel{*}{\rightarrow} \check{\zeta}$ in $L^{\infty}\left(\Gamma_{\mathrm{C}}\right)$ and $\check{\boldsymbol{\pi}}_{\varepsilon} \rightarrow \check{\boldsymbol{\pi}}$ in $W^{1,2}\left(\Gamma_{\mathrm{C}} ; \mathbb{R}^{2}\right)($ in particular, (5.12) and (5.13) are proven). Moreover, since $\check{\zeta}_{\varepsilon}-\zeta_{\varepsilon} \leq 0$, then

$$
\begin{aligned}
\lim _{\varepsilon \rightarrow 0^{+}} \mathrm{R}\left(\check{\zeta}_{\varepsilon}-\zeta_{\varepsilon}, \check{\boldsymbol{\pi}}_{\varepsilon}-\boldsymbol{\pi}_{\varepsilon}\right) & =\lim _{\varepsilon \rightarrow 0^{+}} \int_{\Gamma_{\mathrm{C}}} \mathrm{a}\left(\zeta_{\varepsilon}-\check{\zeta}_{\varepsilon}\right)+\sigma_{\text {yield }}|\check{\boldsymbol{\pi}}-\boldsymbol{\pi}| \mathrm{d} \mathscr{H}^{2} \\
& =\int_{\Gamma_{\mathrm{C}}} \mathrm{a}(\zeta-\check{\zeta})+\sigma_{\text {yield }}|\check{\boldsymbol{\pi}}-\boldsymbol{\pi}| \mathrm{d} \mathscr{H}^{2}=\mathrm{R}(\check{\zeta}-\zeta, \check{\boldsymbol{\pi}}-\boldsymbol{\pi})
\end{aligned}
$$

Thus, to prove (5.14) it sufficies to deal with the convergence of the energies, that is, it is enough to prove that

$$
\limsup _{\varepsilon \rightarrow 0^{+}}\left[\mathrm{E}_{\varepsilon}\left(t_{\varepsilon}, \check{\mathrm{u}}_{\varepsilon}, \check{\zeta}_{\varepsilon}, \check{\boldsymbol{\pi}}_{\varepsilon}\right)-\mathrm{E}_{\varepsilon}\left(t_{\varepsilon}, \mathrm{u}_{\varepsilon}, \zeta_{\varepsilon}, \boldsymbol{\pi}_{\varepsilon}\right)\right] \leq \mathrm{E}_{0}(t, \check{\mathrm{u}}, \check{\zeta}, \check{\boldsymbol{\pi}})-\mathrm{E}_{0}(t, \mathrm{u}, \zeta, \boldsymbol{\pi}) .
$$

The sequence $\check{u}_{\varepsilon}$ can be constructed following what we did in [21] and in the proof of Lemma (3.1) and which we recall also here for convenience of the reader. For fixed $t$ we let

$$
\psi:=-\sum_{\alpha, \beta=1}^{2} \frac{\mathbb{C}_{33 \alpha \beta} \tilde{e}\left(\check{\mathbf{u}}+\mathrm{u}_{\mathrm{D}}(t)\right)_{\alpha \beta}}{\mathbb{C}_{3333}}
$$


and choose $\psi_{\varepsilon} \in C_{0}^{\infty}(\Omega)$ such that $\psi_{\varepsilon} \rightarrow \psi$ and $\varepsilon \partial \psi_{\varepsilon} / \partial x_{\alpha} \rightarrow 0$ in $L^{2}(\Omega)$ for $\alpha=1,2$. Set

$$
\eta_{\varepsilon}\left(x_{1}, x_{2}, x_{3}\right):=\int_{0}^{x_{3}} \psi_{\varepsilon}\left(x_{1}, x_{2}, s\right) \mathrm{d} s
$$

and

$$
\left.\begin{array}{l}
\left(\check{\mathrm{u}}_{\varepsilon}\right)_{\alpha}:=\check{\mathrm{u}}_{\alpha}, \quad \alpha=1,2, \\
\left(\check{\mathrm{u}}_{\varepsilon}\right)_{3}:=\check{\mathrm{u}}_{3}+\varepsilon^{2} \eta_{\varepsilon} .
\end{array}\right\}
$$

In this way we have $\check{\mathrm{u}}_{\varepsilon} \in W^{1,2}\left(\Omega_{1} \cup \Omega_{2} ; \mathbb{R}^{3}\right)$ and $\check{\mathrm{u}}_{\varepsilon} \rightarrow$ ŭ in $W^{1,2}\left(\Omega_{1} \cup \Omega_{2} ; \mathbb{R}^{3}\right)$ (in particular, (5.11) is proven). Since $\mathrm{E}_{0}(t, \check{\mathrm{u}}, \check{\zeta}, \check{\boldsymbol{\pi}})<+\infty$ then for every $\varepsilon$, by construction, $\left(t_{\varepsilon}, \check{\mathrm{u}}_{\varepsilon}, \check{\zeta}_{\varepsilon}, \check{\boldsymbol{\pi}}_{\varepsilon}\right)$ belongs to the effective domain of $E_{\varepsilon}$ and therefore

$$
\begin{aligned}
\mathrm{E}_{\varepsilon}\left(t_{\varepsilon}, \check{\mathrm{u}}_{\varepsilon}, \check{\zeta}_{\varepsilon}, \boldsymbol{\pi}_{\varepsilon}\right)= & \frac{1}{2} \int_{\Omega_{1} \cup \Omega_{2}} \mathbb{C} e^{\varepsilon}\left(\check{\mathrm{u}}_{\varepsilon}+\mathrm{u}_{\mathrm{D}}\left(t_{\varepsilon}\right)\right): e^{\varepsilon}\left(\check{\mathrm{u}}_{\varepsilon}+\mathrm{u}_{\mathrm{D}}\left(t_{\varepsilon}\right)\right)-2 \mathrm{f}\left(t_{\varepsilon}\right) \cdot\left(\check{\mathrm{u}}_{\varepsilon}+\mathrm{u}_{\mathrm{D}}\left(t_{\varepsilon}\right)\right) \mathrm{d} x \\
& +\int_{\Gamma_{\mathrm{C}}} \check{\zeta}_{\varepsilon} \mathrm{Q}^{\varepsilon}\left(\llbracket \check{\mathrm{u}}_{\varepsilon} \rrbracket-\mathbb{T} \check{\boldsymbol{\pi}}_{\varepsilon}\right)+\frac{\kappa_{\mathrm{H}}}{2}\left|\check{\boldsymbol{\pi}}_{\varepsilon}\right|^{2}+\frac{\kappa_{0}}{2}\left|\nabla_{\mathrm{S}} \check{\boldsymbol{\pi}}_{\varepsilon}\right|^{2} \mathrm{~d} \mathscr{H}^{2} .
\end{aligned}
$$

By (3.2) and since $\check{u}$ and $\mathrm{u}_{\mathrm{D}}$ are in $W_{\mathrm{KL}}^{1,2}\left(\Omega_{1} \cup \Omega_{2} ; \mathbb{R}^{3}\right)$, we have

$$
e^{\varepsilon}\left(\check{\mathbf{u}}_{\varepsilon}+\mathbf{u}_{\mathbf{D}}\left(t_{\varepsilon}\right)\right)=\left(\begin{array}{cc}
e\left(\check{\mathbf{u}}+\mathrm{u}_{\mathrm{D}}\left(t_{\varepsilon}\right)\right)_{\alpha \beta} & \frac{\varepsilon}{2} \frac{\partial \eta_{\varepsilon}}{\partial x_{\alpha}} \\
\operatorname{sym} & \psi_{\varepsilon}
\end{array}\right) .
$$

Taking the limit as $\varepsilon \rightarrow 0^{+}$we have

$$
\lim _{\varepsilon \rightarrow 0^{+}}\left\|e^{\varepsilon}\left(\check{\mathbf{u}}_{\varepsilon}+\mathrm{u}_{\mathrm{D}}\left(t_{\varepsilon}\right)\right)-\left(\begin{array}{cc}
\tilde{e}\left(\check{\mathbf{u}}+\mathrm{u}_{\mathrm{D}}(t)\right) & 0 \\
0 & -\sum_{\alpha, \beta=1}^{2} \mathbb{C}_{33 \alpha \beta} \tilde{e}\left(\check{\mathbf{u}}+\mathbf{u}_{\mathrm{D}}(t)\right)_{\alpha \beta} / \mathbb{C}_{3333}
\end{array}\right)\right\|_{L^{2}\left(\Omega_{1} \cup \Omega_{2} ; \mathbb{R}^{3 \times 3}\right)}=0,
$$

and therefore, concerning the bulk part in (5.19), we obtain

$$
\lim _{\varepsilon \rightarrow 0^{+}} \int_{\Omega_{1} \cup \Omega_{2}} \mathbb{C} e^{\varepsilon}\left(\check{\mathbf{u}}_{\varepsilon}+\mathbf{u}_{\mathbf{D}}\left(t_{\varepsilon}\right)\right): e^{\varepsilon}\left(\check{\mathbf{u}}_{\varepsilon}+\mathbf{u}_{\mathbf{D}}\left(t_{\varepsilon}\right)\right) \mathrm{d} x=\int_{\Omega_{1} \cup \Omega_{2}} \mathbb{C}^{0} \tilde{e}\left(\check{\mathbf{u}}+\mathbf{u}_{\mathrm{D}}(t)\right): \tilde{e}\left(\check{\mathbf{u}}+\mathbf{u}_{\mathbf{D}}(t)\right) \mathrm{d} x,
$$

and, by the lower semicontinuity Lemma 5.1, we have

$$
\begin{gathered}
\limsup _{\varepsilon \rightarrow 0^{+}}\left[\int_{\Omega_{1} \cup \Omega_{2}} \mathbb{C} e^{\varepsilon}\left(\check{\mathbf{u}}_{\varepsilon}+\mathbf{u}_{\mathrm{D}}\left(t_{\varepsilon}\right)\right): e^{\varepsilon}\left(\check{\mathbf{u}}_{\varepsilon}+\mathbf{u}_{\mathrm{D}}\left(t_{\varepsilon}\right)\right) \mathrm{d} x-\int_{\Omega_{1} \cup \Omega_{2}} \mathbb{C} e^{\varepsilon}\left(\mathbf{u}_{\varepsilon}+\mathbf{u}_{\mathrm{D}}\left(t_{\varepsilon}\right)\right): e^{\varepsilon}\left(\mathbf{u}_{\varepsilon}+\mathbf{u}_{\mathbf{D}}\left(t_{\varepsilon}\right)\right) \mathrm{d} x\right] \\
\leq \int_{\Omega_{1} \cup \Omega_{2}} \mathbb{C} \tilde{e}\left(\check{\mathbf{u}}+\mathbf{u}_{\mathrm{D}}(t)\right): \tilde{e}\left(\check{\mathbf{u}}+\mathbf{u}_{\mathrm{D}}(t)\right) \mathrm{d} x-\int_{\Omega_{1} \cup \Omega_{2}} \mathbb{C}\left(\mathbf{u}+\mathbf{u}_{\mathrm{D}}(t)\right): \tilde{e}\left(\mathbf{u}+\mathbf{u}_{\mathbf{D}}(t)\right) \mathrm{d} x .
\end{gathered}
$$

By the same argument used to prove (5.10) we have

$$
\lim _{\varepsilon \rightarrow 0^{+}} \int_{\Gamma_{\mathrm{C}}} \zeta_{\varepsilon} \mathrm{Q}^{\varepsilon}\left(\llbracket \mathrm{u}_{\varepsilon} \rrbracket-\mathbb{T} \boldsymbol{\pi}_{\varepsilon}\right) \mathrm{d} \mathscr{H}^{2}=\int_{\Gamma_{\mathrm{C}}} \zeta \mathrm{Q}^{0}(\llbracket \mathrm{u} \rrbracket-\mathbb{T} \boldsymbol{\pi}) \mathrm{d} \mathscr{H}^{2}
$$

and

$$
\lim _{\varepsilon \rightarrow 0^{+}} \int_{\Gamma_{\mathrm{C}}} \check{\zeta}_{\varepsilon} \mathrm{Q}^{\varepsilon}\left(\llbracket \check{\mathrm{u}}_{\varepsilon} \rrbracket-\mathbb{T} \check{\boldsymbol{\pi}}_{\varepsilon}\right) \mathrm{d} \mathscr{H}^{2}=\int_{\Gamma_{\mathrm{C}}} \check{\zeta} \mathrm{Q}^{0}(\llbracket \check{u} \rrbracket-\mathbb{T} \check{\pi}) \mathrm{d} \mathscr{H}^{2} .
$$

Since $\boldsymbol{\pi}_{\varepsilon} \rightarrow \boldsymbol{\pi}$ and $\check{\boldsymbol{\pi}}_{\varepsilon} \rightarrow \check{\boldsymbol{\pi}}$ then

$$
\lim _{\varepsilon \rightarrow 0^{+}} \int_{\Gamma_{\mathrm{C}}} \frac{\kappa_{\mathrm{H}}}{2}\left|\pi_{\varepsilon}\right|^{2} \mathrm{~d} \mathscr{H}^{2}=\int_{\Gamma_{\mathrm{C}}} \frac{\kappa_{\mathrm{H}}}{2}|\pi|^{2} \mathrm{~d} \mathscr{H}^{2} \quad \text { and } \quad \lim _{\varepsilon \rightarrow 0^{+}} \int_{\Gamma_{\mathrm{C}}} \frac{\kappa_{\mathrm{H}}}{2}\left|\check{\pi}_{\varepsilon}\right|^{2} \mathrm{~d} \mathscr{H}^{2}=\int_{\Gamma_{\mathrm{C}}} \frac{\kappa_{\mathrm{H}}}{2}|\check{\pi}|^{2} \mathrm{~d} \mathscr{H}^{2}
$$

Concerning the term involving the gradient, by the so-called binomial trick we have

$$
\left|\nabla_{\mathrm{S}} \check{\boldsymbol{\pi}}_{\varepsilon}\right|^{2}-\left|\nabla_{\mathrm{S}} \boldsymbol{\pi}_{\varepsilon}\right|^{2}=\left(\nabla_{\mathrm{S}} \check{\boldsymbol{\pi}}_{\varepsilon}-\nabla_{\mathrm{S}} \boldsymbol{\pi}_{\varepsilon}\right) \cdot\left(\nabla_{\mathrm{S}} \check{\boldsymbol{\pi}}_{\varepsilon}+\nabla_{\mathrm{S}} \boldsymbol{\pi}_{\varepsilon}\right)=\left(\nabla_{\mathrm{S}} \check{\boldsymbol{\pi}}-\nabla_{\mathrm{S}} \boldsymbol{\pi}\right) \cdot\left(\nabla_{\mathrm{S}} \check{\boldsymbol{\pi}}_{\varepsilon}+\nabla_{\mathrm{S}} \boldsymbol{\pi}_{\varepsilon}\right)
$$


and since $\nabla_{\mathrm{S}} \check{\boldsymbol{\pi}}_{\varepsilon}+\nabla_{\mathrm{S}} \boldsymbol{\pi}_{\varepsilon} \rightarrow \nabla_{\mathrm{S}} \check{\boldsymbol{\pi}}+\nabla_{\mathrm{S}} \boldsymbol{\pi}$ in $L^{2}\left(\Gamma_{\mathrm{C}} ; \mathbb{R}^{2}\right)$, then we get

$$
\lim _{\varepsilon \rightarrow 0^{+}} \int_{\Gamma_{\mathrm{C}}}\left(\left|\nabla_{\mathrm{S}} \check{\pi}_{\varepsilon}\right|^{2}-\left|\nabla_{\mathrm{S}} \pi_{\varepsilon}\right|^{2}\right) \mathrm{d} \mathscr{H}^{2}=\int_{\Gamma_{\mathrm{C}}}\left(\left|\nabla_{\mathrm{S}} \check{\pi}\right|^{2}-\left|\nabla_{\mathrm{S}} \pi\right|^{2}\right) \mathrm{d} \mathscr{H}^{2}
$$

and therefore

$$
\lim _{\varepsilon \rightarrow 0^{+}} \int_{\Gamma_{\mathrm{C}}} \frac{\kappa_{0}}{2}\left|\nabla_{\mathrm{S}} \check{\boldsymbol{\pi}}_{\varepsilon}\right|^{2} \mathrm{~d} \mathscr{H}^{2}-\int_{\Gamma_{\mathrm{C}}} \frac{\kappa_{0}}{2}\left|\nabla_{\mathrm{S}} \boldsymbol{\pi}_{\varepsilon}\right|^{2} \mathrm{~d} \mathscr{H}^{2}=\int_{\Gamma_{\mathrm{C}}} \frac{\kappa_{0}}{2}\left|\nabla_{\mathrm{S}} \check{\pi}\right|^{2} \mathrm{~d} \mathscr{H}^{2}-\int_{\Gamma_{\mathrm{C}}} \frac{\kappa_{0}}{2}\left|\nabla_{\mathrm{S}} \pi\right|^{2} \mathrm{~d} \mathscr{H}^{2} .
$$

Inequality (5.16) follows by (5.20), (5.21), (5.22), (5.23), and Lemma 3.1. Using moreover (5.24) we finally obtain also (5.14) and the proof is concluded.

\subsection{Convergence of solutions}

The aim of this section is to show, assuming $q_{\varepsilon}^{0} \rightarrow q^{0}$ in $\mathcal{Q}$, the convergences of (the solutions to) the corresponding quasi-static evolution problems. The notation in use is that of [50]. According to what we have done above, we denote by $\mathcal{Q}$ the topological product of the spaces $\mathcal{U}$ and $\mathcal{Z}$ endowed, respectively, with the weak and the weak* topology. Since $\mathcal{U}$ is reflexive here, its weak topology is also weak*, and thus the convergence in $\mathcal{Q}$ will be denoted simply by $\stackrel{*}{\rightarrow}$. Setting $\mathrm{q}=(\mathrm{u}, z)$ and $z=(\zeta, \boldsymbol{\pi})$, we shall write, for instance, $(t, \mathbf{q})$ in place of $(t, \mathbf{u}, z)$ and the latter in place of $(t, \mathbf{u}, \zeta, \boldsymbol{\pi})$.

Our results will be achieved by applying general abstract theorems proven in $[19,44,50]$. In what follows we write and check the assumptions needed to apply those theorems.

The dissipation distance

$$
\mathcal{D}\left(z_{1}, z_{2}\right)=\mathrm{R}\left(z_{2}-z_{1}\right)
$$

satisfies the following properties, corresponding to [50, Formulas (2.2)-(2.4)].

Pseudo distance:

$$
\mathcal{D}\left(z_{1}, z_{1}\right)=0 \text { and } \mathcal{D}\left(z_{1}, z_{3}\right) \leq \mathcal{D}\left(z_{1}, z_{2}\right)+\mathcal{D}\left(z_{2}, z_{3}\right) \text { for any } z_{1}, z_{2}, z_{3} \in \mathcal{Z} .
$$

Lower semi-continuity of $\mathcal{D}$ :

$$
\mathcal{D}: z \times z \rightarrow[0,+\infty] \text { is } \mathrm{w}^{*} \text {-lower semi-continuous. }
$$

Positivity of $\mathcal{D}$ :

if a sequence $\left(z_{n}\right)$ in $z$ and $z \in Z$ are such that $\min \left\{\mathcal{D}\left(z_{n}, z\right), \mathcal{D}\left(z, z_{n}\right)\right\} \rightarrow 0$, then $z_{n} \rightarrow z$ weakly* in $z$.

From (5.26) it follows (2.5) of [50], that is

Lower $\Gamma$-limit for $\mathcal{D}$ :

for any pair of stable sequences $\left(t_{n}, \mathbf{q}_{n}\right),\left(\check{t}_{n}, \check{\mathbf{q}}_{n}\right)$ such that $\left(t_{n}, \mathbf{q}_{n}\right) \stackrel{*}{\rightarrow}(t, \mathbf{q}),\left(\check{t}_{n}, \check{\mathbf{q}}_{n}\right) \stackrel{*}{\rightarrow}(\check{t}, \check{\mathbf{q}})$ in $[0, T] \times \mathcal{Q}$, we have

$\mathcal{D}(z, \check{z}) \leq \liminf _{n \rightarrow+\infty} \mathcal{D}\left(z_{n}, \check{z}_{n}\right)$.

From Korn's inequality, (2.4), (3.4) and (3.5), we find

$$
\begin{aligned}
\mathrm{E}_{\varepsilon}(t, \mathbf{u}, z) & \geq \frac{1}{2} \int_{\Omega_{1} \cup \Omega_{2}} \mathbb{C} e^{\varepsilon}\left(\mathbf{u}+\mathrm{u}_{\mathrm{D}}(t)\right): e^{\varepsilon}\left(\mathbf{u}+\mathrm{u}_{\mathrm{D}}(t)\right)-2 \mathbf{f}^{\varepsilon}(t) \cdot\left(\mathbf{u}+\mathrm{u}_{\mathrm{D}}(t)\right) \mathrm{d} x \\
& \geq c\left\|e\left(\mathbf{u}+\mathbf{u}_{\mathrm{D}}(t)\right)\right\|_{L^{2}}^{2}-\frac{1}{2 \alpha}\|\mathrm{f}(t)\|_{L^{2}}^{2}-\frac{\alpha}{2}\left\|\mathbf{u}+\mathbf{u}_{\mathrm{D}}(t)\right\|_{L^{2}}^{2} \geq K\|\mathbf{u}\|_{W^{1,2}}^{2}-C,
\end{aligned}
$$

where $f=\left(f_{1}, f_{2}, f_{3}\right)$, see (3.3). This last inequality, together with a similar computation for $E_{0}$, shows that the set $\bigcup_{\varepsilon \geq 0}\left\{q \in \mathcal{Q}: \mathrm{E}_{\varepsilon}(t, q) \leq E\right\}$ is weakly relatively compact. Moreover, for any $\varepsilon \geq 0$ the functionals $\mathrm{E}_{\varepsilon}(t, \cdot)$ are weakly lower semicontinuous in $\mathcal{Q}$ due to the convexity of the bulk part of the energy and Lemma 3.1. Thus the sublevels are also closed and the following property (corresponding to (2.6) of [50]) holds 
Compactness of energy sublevels:

for all $t \in[0, T]$ and all $E \in \mathbb{R}$ we have

(i) $\quad\left\{\mathbf{q} \in \mathcal{Q}: \mathrm{E}_{\varepsilon}(t, \mathbf{q}) \leq E\right\}$ is compact for any $\varepsilon \geq 0$;

(ii) $\bigcup_{\varepsilon \geq 0}\left\{\mathbf{q} \in \mathcal{Q}: \mathrm{E}_{\varepsilon}(t, \mathbf{q}) \leq E\right\}$ is relatively compact.

Since $f$ and $w$ are continuously differentiable with respect to $t$ (see Section 3.1), then $E_{\varepsilon}(\cdot, q) \in$ $C^{1}([0, T])$ for all $\varepsilon \geq 0$ and all $\mathrm{q} \in \mathcal{Q}$ for which $\mathrm{E}_{\varepsilon}(\cdot, \mathrm{q})<+\infty$.

If $\mathrm{E}_{\varepsilon}(s, \mathrm{u}, z)<+\infty$ for $s \in[0, T]$, then we have

$$
\partial_{t} \mathrm{E}_{\varepsilon}(t, \mathbf{u}, z)=\int_{\Omega_{1} \cup \Omega_{2}} \mathbb{C} e^{\varepsilon}\left(\dot{\mathbf{u}}_{\mathrm{D}}(t)\right): e^{\varepsilon}\left(\mathbf{u}+\mathrm{u}_{\mathrm{D}}(t)\right)-\dot{\mathrm{f}}^{\varepsilon}(t) \cdot\left(\mathbf{u}+\mathrm{u}_{\mathrm{D}}(t)\right)-\mathbf{f}^{\varepsilon}(t) \cdot \dot{\mathrm{u}}_{\mathrm{D}}(t) \mathrm{d} x
$$

and, since $\left(e\left(\dot{\mathbf{u}}_{\mathrm{D}}\right)\right)_{i 3}=0$ (see (3.5)), by inequality (5.29) we have

$$
\left|\partial_{t} \mathrm{E}_{\varepsilon}(t, \mathrm{u}, z)\right| \leq c\left(\left\|e\left(\dot{\mathrm{u}}_{\mathrm{D}}\right)(t)\right\|_{L^{2}}^{2}+\|\dot{\mathrm{f}}(t)\|_{L^{2}}^{2}+\left\|\mathrm{u}_{\mathrm{D}}(t)\right\|_{L^{2}}^{2}+\|\mathrm{f}(t)\|_{L^{2}}^{2}+\left\|\dot{\mathrm{u}}_{\mathrm{D}}(t)\right\|_{L^{2}}^{2}+\mathrm{E}_{\varepsilon}(t, \mathrm{u}, z)+C\right),
$$

which, together with a similar computation for $\mathrm{E}_{0}$, leads to (see (2.7) of [50])

Uniform control of the power $\partial_{t} \mathrm{E}_{\varepsilon}$ :

$$
\begin{aligned}
& \text { there exist } c_{0}^{E} \in \mathbb{R} \text { and } c_{1}^{E}>0 \text { such that } \\
& \text { for any } \varepsilon \geq 0, t \in[0, T] \text { and } \mathbf{q} \in \mathcal{Q} \text {, } \\
& \text { if } \mathrm{E}_{\varepsilon}(t, \mathbf{q})<+\infty \text { then } \mathrm{E}_{\varepsilon}(\cdot, \mathbf{q}) \in C^{1}([0, T]) \text { and } \\
& \left|\partial_{t} \mathrm{E}_{\varepsilon}(s, \mathbf{q})\right| \leq c_{1}^{E}\left(c_{0}^{E}+\mathrm{E}_{\varepsilon}(s, \mathbf{q})\right) \text { for all } s \in[0, T] .
\end{aligned}
$$

By definition of $\mathrm{E}_{0}$ (given in (3.17)) it follows that if $\mathrm{E}_{0}(0, \mathrm{u}, z)$ is finite then $\mathrm{u} \in W_{\mathrm{KL}}^{1,2}\left(\Omega_{1} \cup \Omega_{2} ; \mathbb{R}^{3}\right)$ and thus $\mathrm{E}_{0}(t, \mathrm{u}, z)$ is finite for every $t \in[0, T]$. Since $\mathrm{f}$ and $\mathbf{w}$ are $C^{1}$ with respect to $t$, condition $(2.8)$ of $[50]$ is satisfied, namely

Uniform time-continuity of the power $\partial_{t} \mathrm{E}_{0, \kappa}$ :

$$
\begin{aligned}
& \text { for every } \eta>0 \text { and } E \in \mathbb{R} \text { there exists } \delta>0 \text { such that } \\
& \mathrm{E}_{0}(0, \mathrm{q}) \leq E,\left|t_{1}-t_{2}\right|<\delta \Rightarrow\left|\partial_{t} \mathrm{E}_{0}\left(t_{1}, \mathrm{q}\right)-\partial_{t} \mathrm{E}_{0}\left(t_{2}, \mathrm{q}\right)\right|<\eta .
\end{aligned}
$$

By Lemma 5.1 we get the following property (2.10) of [50]

Lower $\Gamma$-limit for $\mathrm{E}_{\varepsilon, \kappa}$ :

for any uniformly stable sequence $\left(t_{\varepsilon}, \mathrm{q}_{\varepsilon}\right)$ such that

$\left(t_{\varepsilon}, \mathbf{q}_{\varepsilon}\right) \stackrel{*}{\rightarrow}(t, \mathbf{q})$ in $[0, T] \times \mathcal{Q}$, we have

$\mathrm{E}_{0}(t, \mathbf{q}) \leq \liminf _{\varepsilon \rightarrow 0^{+}} \mathrm{E}_{\varepsilon}\left(t_{\varepsilon}, \mathrm{q}_{\varepsilon}\right)$.

The following lemma essentially corresponds to property (2.9) of [50]; the only difference is that we establish the convergence in the open interval $(0, T)$ instead of its closure. This fact will not affect the arguments used in the sequel.

Lemma 5.3 (Conditioned continuous convergence of the power). Let $(t, \mathbf{q}) \in(0, T) \times \mathcal{Q}$ and let $\left(t_{\varepsilon}, \mathbf{q}_{\varepsilon}\right)$ be a uniformly stable sequence. If $\left(t_{\varepsilon}, \mathrm{q}_{\varepsilon}\right) \stackrel{*}{\rightarrow}(t, \mathbf{q})$ in $[0, T] \times \mathcal{Q}$, then

$$
\partial_{t} \mathrm{E}_{\varepsilon}\left(t_{\varepsilon}, \mathbf{q}_{\varepsilon}\right) \rightarrow \partial_{t} \mathrm{E}_{0}(t, \mathbf{q}) .
$$

Proof. Since $\partial_{t} \mathrm{E}_{\varepsilon}=\partial_{t} \overline{\mathrm{E}}_{\varepsilon}$ for every $\varepsilon \geq 0$, where $\overline{\mathrm{E}}_{\varepsilon}$ has been defined in (5.15), then we can equivalently prove that

$$
\partial_{t} \overline{\mathrm{E}}_{\varepsilon}\left(t_{\varepsilon}, \mathbf{q}_{\varepsilon}\right) \rightarrow \partial_{t} \overline{\mathrm{E}}_{0}(t, \mathbf{q})
$$

The proof can now be done by following exactly the same arguments of the proof of Lemma 5.2 of [21], starting from the convergence property stated in the following lemma. 
Lemma 5.4. Let $(t, \mathrm{q}) \in(0, T) \times \mathcal{Q}$ and let $\left(t_{\varepsilon}, \mathrm{q}_{\varepsilon}\right)$ be a uniformly stable sequence w.r. to $\left(\mathrm{E}_{\varepsilon}\right)$ and $\left(S_{\varepsilon}\right)$. If $\left(t_{\varepsilon}, \mathrm{q}_{\varepsilon}\right) \stackrel{*}{\rightarrow}(t, \mathrm{q})$ in $[0, T] \times \mathcal{Q}$, then

$$
\overline{\mathrm{E}}_{\varepsilon}\left(t_{\varepsilon}, \mathrm{q}_{\varepsilon}\right) \rightarrow \overline{\mathrm{E}}_{0}(t, \mathrm{q}) \quad \text { as } \quad \varepsilon \rightarrow 0^{+} .
$$

Proof. Let $\mathrm{q}=:(\mathrm{u}, \zeta, \boldsymbol{\pi})$ and $\mathrm{q}_{\varepsilon}=:\left(\mathrm{u}_{\varepsilon}, \zeta_{\varepsilon}, \boldsymbol{\pi}_{\varepsilon}\right)$. Being uniformly stable with respect to $\left(\mathrm{E}_{\varepsilon}\right)$ and $\left(\mathrm{S}_{\varepsilon}\right)$, the sequence $\left(t_{\varepsilon}, \mathrm{q}_{\varepsilon}\right)$ satisfies the assumptions of Lemma 5.2 which we apply now with the choice $\mathrm{u}=\mathrm{u}, \breve{\zeta}=\zeta$ and $\check{\boldsymbol{\pi}}=\boldsymbol{\pi}$. Hence there exist $\check{\mathrm{q}}_{\varepsilon}=\left(\check{\mathrm{u}}_{\varepsilon}, \check{z}_{\varepsilon}\right)=\left(\check{\mathrm{u}}_{\varepsilon}, \check{\zeta}_{\varepsilon}, \check{\boldsymbol{\pi}}_{\varepsilon}\right) \in \mathcal{Q}$ with $\check{\boldsymbol{\pi}}_{\varepsilon}=\boldsymbol{\pi}_{\varepsilon}-\check{\boldsymbol{\pi}}-\boldsymbol{\pi}=\boldsymbol{\pi}_{\varepsilon}$ such that

$$
\begin{aligned}
& \check{\mathrm{q}}_{\varepsilon} \stackrel{*}{\rightarrow} \mathrm{q} \text { in } \mathcal{Q}, \\
& \underset{\varepsilon \rightarrow 0^{+}}{\limsup }\left[\overline{\mathrm{E}}_{\varepsilon}\left(t_{\varepsilon}, \check{\mathrm{q}}_{\varepsilon}\right)+\mathrm{R}\left(\check{\mathrm{q}}_{\varepsilon}-\mathrm{q}_{\varepsilon}\right)\right] \leq \overline{\mathrm{E}}_{0}(t, \mathrm{q}) .
\end{aligned}
$$

By the stability of $\left(t_{\varepsilon}, \mathrm{q}_{\varepsilon}\right)$ with respect to $\left(\mathrm{E}_{\varepsilon}\right)$ and $\left(\mathrm{S}_{\varepsilon}\right)$ we have

$$
\begin{aligned}
\overline{\mathrm{E}}_{\varepsilon}\left(t_{\varepsilon}, \mathrm{q}_{\varepsilon}\right)+\int_{\Gamma_{\mathrm{C}}} \frac{\kappa_{0}}{2}\left|\nabla_{\mathrm{S}} \boldsymbol{\pi}_{\varepsilon}\right|^{2} \mathrm{~d} \mathscr{H}^{2} & \leq \overline{\mathrm{E}}_{\varepsilon}\left(t_{\varepsilon}, \check{\mathrm{q}}_{\varepsilon}\right)+\int_{\Gamma_{\mathrm{C}}} \frac{\kappa_{0}}{2}\left|\nabla_{\mathrm{S}} \check{\boldsymbol{\pi}}_{\varepsilon}\right|^{2} \mathrm{~d} \mathscr{H}^{2}+\mathrm{R}\left(\check{\mathrm{q}}_{\varepsilon}-\mathrm{q}_{\varepsilon}\right) \\
& =\overline{\mathrm{E}}_{\varepsilon}\left(t_{\varepsilon}, \check{\mathrm{q}}_{\varepsilon}\right)+\int_{\Gamma_{\mathrm{C}}} \frac{\kappa_{0}}{2}\left|\nabla_{\mathrm{S}} \boldsymbol{\pi}_{\varepsilon}\right|^{2} \mathrm{~d} \mathscr{H}^{2}+\mathrm{R}\left(\check{\mathrm{q}}_{\varepsilon}-\mathrm{q}_{\varepsilon}\right)
\end{aligned}
$$

from which we get

$$
\overline{\mathrm{E}}_{\varepsilon}\left(t_{\varepsilon}, \mathrm{q}_{\varepsilon}\right) \leq \overline{\mathrm{E}}_{\varepsilon}\left(t_{\varepsilon}, \check{\mathrm{q}}_{\varepsilon}\right)+\mathrm{R}\left(\check{\mathrm{q}}_{\varepsilon}-\mathrm{q}_{\varepsilon}\right)
$$

which, together with (5.36) gives

$$
\limsup _{\varepsilon \rightarrow 0^{+}} \overline{\mathrm{E}}_{\varepsilon}\left(t_{\varepsilon}, \mathrm{q}_{\varepsilon}\right) \leq \overline{\mathrm{E}}_{0}(t, \mathbf{q})
$$

Hence (5.35) follows from the inequality above and an adaptation of Lemma 5.1 to $\overline{\mathrm{E}}_{\varepsilon}$.

By [50, Proposition 2.2] we get that Lemma 5.2 implies property (2.11) of [50], namely

Conditioned upper-semicontinuity of stable sets:

- for any uniformly stable sequence $\left(t_{\varepsilon}, \mathrm{q}_{\varepsilon}\right)$ w. r. to $\left(\mathrm{E}_{\varepsilon}\right)$ and $\left(\mathrm{S}_{\varepsilon}\right)$ such that $\left(t_{\varepsilon}, \mathrm{q}_{\varepsilon}\right) \stackrel{*}{\rightarrow}(t, \mathbf{q})$ in $[0, T] \times \mathcal{Q}$ we have that $\mathbf{q} \in \mathrm{S}_{0}(t)$.

The existence of energetic solutions associated with the functionals $\mathrm{E}_{\varepsilon}$ and $\mathrm{R}$, for fixed $\varepsilon>0$, has been proven in [60]. An alternative proof could be made as in [21, Theorem 5.4], based on a general existence theorem of Mielke [46], see also [19,44,50].

We are now in a position to state our main dimension reduction result.

Theorem 5.5 (Convergence as $\varepsilon \rightarrow 0)$. Let $\mathrm{q}_{0}=\left(\mathrm{u}_{0}, z_{0}\right) \in \mathcal{Q}$ and, for any $\varepsilon>0, \mathrm{q}_{0, \varepsilon}=\left(\mathrm{u}_{0, \varepsilon}, z_{0, \varepsilon}\right) \in$ $\mathrm{S}_{\varepsilon}(0)$ with $\mathrm{q}_{0, \varepsilon} \stackrel{*}{\rightarrow} \mathrm{q}_{0}$ and $\mathrm{E}_{\varepsilon}\left(0, \mathrm{q}_{0, \varepsilon}\right) \rightarrow \mathrm{E}_{0}\left(0, \mathrm{q}_{0}\right)$ as $\varepsilon \rightarrow 0$, let further $\mathrm{q}_{\varepsilon}=\left(\mathrm{u}_{\varepsilon}, z_{\varepsilon}\right):[0, T] \rightarrow \mathcal{Q}$ be an energetic solution to the problem $\left(\mathcal{Q}, \mathrm{E}_{\varepsilon}, \mathrm{R}, \mathrm{q}_{\varepsilon}(0)\right)$. Then there exist $\mathrm{q}=(\mathrm{u}, z):[0, T] \rightarrow \mathcal{Q}$ such that, up to a subsequence,

$$
\begin{aligned}
& \mathrm{E}_{\varepsilon}\left(t, \mathrm{q}_{\varepsilon}(t)\right) \rightarrow \mathrm{E}_{0}(t, \mathrm{q}(t)) \\
& \mathrm{R}\left(z_{\varepsilon}(t)-z_{\varepsilon}(0)\right) \rightarrow \mathrm{R}(z(t)-z(0)) \\
& \partial_{t} \mathrm{E}_{\varepsilon}\left(\cdot, \mathrm{q}_{\varepsilon}(\cdot)\right) \rightarrow \partial_{t} \mathrm{E}_{0}(\cdot, \mathrm{q}(\cdot)) \\
& \zeta_{\varepsilon}(t) \stackrel{*}{\rightarrow} \zeta(t) \\
& \boldsymbol{\pi}_{\varepsilon}(t) \rightarrow \boldsymbol{\pi}(t) \\
& \mathrm{u}_{\varepsilon}(t) \rightarrow \mathrm{u}(t)
\end{aligned}
$$$$
\text { for every } t \in[0, T] \text {, }
$$$$
\text { for every } t \in[0, T] \text {, }
$$$$
\text { in } L^{1}(0, T) \text {, }
$$$$
\text { in } L^{\infty}\left(\Gamma_{\mathrm{C}}\right) \text { for every } t \in[0, T] \text {, }
$$$$
\text { in } W^{1,2}\left(\Gamma_{\mathrm{C}} ; \mathbb{R}^{2}\right) \text { for every } t \in[0, T] \text {, }
$$$$
\text { in } W^{1,2}\left(\Omega_{1} \cup \Omega_{2} ; \mathbb{R}^{3}\right) \text { for every } t \in[0, T] \text {. }
$$

Moreover, any $\mathrm{q}$ obtained in this way is an energetic solution to the problem $\left(\mathcal{U} \times \mathcal{Z}, \mathrm{E}_{0}, \mathrm{R}, \mathrm{q}_{0}\right)$. 
Proof. Since $t \mapsto \mathrm{q}_{\varepsilon}(t)=\left(\mathrm{u}_{\varepsilon}(t), z_{\varepsilon}(t)\right)$ is an energetic solution and the power is controlled uniformly in $\varepsilon$, cf. (5.32), by a Gronwall-inequality argument, it can be shown (see $[19,44,50]$ ) that for every $t \in[0, T]$ the sequence $\mathrm{E}_{\varepsilon}\left(t, \mathrm{u}_{\varepsilon}(t), z_{\varepsilon}(t)\right)$ is bounded uniformly in $\varepsilon$; hence in particular $\left(t, q_{\varepsilon}\right)$ is a uniformly stable sequence. Hence, from the uniform coercivity of $\mathrm{E}_{\varepsilon}(t, \cdot, \cdot)$ and Korn's inequality (see (5.29)), we have that, for every $t \in[0, T]$

$$
\begin{aligned}
& \left(\boldsymbol{\pi}_{\varepsilon}(t)\right) \text { is uniformly bounded in } W^{1,2}\left(\Gamma_{\mathrm{C}} ; \mathbb{R}^{2}\right), \\
& \left(\mathbf{u}_{\varepsilon}(t)\right) \text { is uniformly bounded in } W^{1,2}\left(\Omega_{1} \cup \Omega_{2} ; \mathbb{R}^{3}\right),
\end{aligned}
$$

and, but this is trivial since $\zeta_{\varepsilon} \in[0,1]$,

$$
\left(\zeta_{\varepsilon}(t)\right) \text { is uniformly bounded in } L^{\infty}\left(\Gamma_{\mathrm{C}}\right) \text {. }
$$

By applying a Helly's type theorem (namely [50, Theorem A.1]) to the sequence $\left(z_{\varepsilon}\right)=\left(\zeta_{\varepsilon}, \boldsymbol{\pi}_{\varepsilon}\right)$, we have that there exist $\zeta \in L^{\infty}\left(\Gamma_{\mathrm{C}}\right)$ and $\pi \in W^{1,2}\left(\Gamma_{\mathrm{C}} ; \mathbb{R}^{2}\right)$ such that, up to a subsequence (not relabeled)

$$
\begin{aligned}
& \zeta_{\varepsilon}(t) \stackrel{*}{\rightarrow} \zeta(t) \quad \text { in } L^{\infty}\left(\Gamma_{\mathrm{C}}\right), \\
& \boldsymbol{\pi}_{\varepsilon}(t) \stackrel{*}{\rightarrow} \boldsymbol{\pi}(t) \quad \text { in } W^{1,2}\left(\Gamma_{\mathrm{C}} ; \mathbb{R}^{2}\right)
\end{aligned}
$$

for every $t \in[0, T]$. We have thus proven $(5.38 \mathrm{~d})$ and the weak convergence in $(5.38 \mathrm{e})$.

Moreover, for every $t \in[0, T]$ there exists a subsequence $\left(\varepsilon^{t}\right)$ such that

$$
\mathrm{u}_{\varepsilon^{t}}(t) \rightarrow \mathrm{u}(t) \quad \text { in } W^{1,2}\left(\Omega_{1} \cup \Omega_{2} ; \mathbb{R}^{3}\right),
$$

and

$$
\theta(t):=\limsup _{\varepsilon \rightarrow 0^{+}} \partial_{t} \mathrm{E}_{\varepsilon}\left(t, \mathrm{q}_{\varepsilon}(t)\right)=\lim _{\varepsilon^{t} \rightarrow 0} \partial_{t} \mathrm{E}_{\varepsilon^{t}}\left(t, \mathrm{q}_{\varepsilon^{t}}(t)\right) .
$$

From $(5.39),(5.40),(5.41),(5.42)$ and Lemma 5.3 it follows that

$$
\theta(t)=\partial_{t} \mathrm{E}_{0}(t, \mathrm{u}(t), z(t))
$$

from which $(5.38 \mathrm{c})$ follows. By [50, Theorem 3.1] and its proof we deduce that $\mathrm{q}(t):=(\mathrm{u}(t), z(t))$ is an energetic solution to the problem $\left(\mathcal{U} \times \mathcal{Z}, \mathrm{E}_{0}, \mathrm{R}, \mathrm{q}(0)\right)$. By the stability inequality for the limit problem and the strict convexity of the map $\mathrm{u} \mapsto \mathrm{E}_{0}(t, \mathrm{u}, z)$, the function $\mathrm{u}$ is uniquely determined by $z$. Hence the convergence in (5.41) holds for the whole (sub)sequence $\varepsilon$, that is (5.38f).

Let us prove $(5.38 \mathrm{~b})$. Since $\left(\mathrm{u}_{\varepsilon}, z_{\varepsilon}\right)$ is an energetic solution, from the energy balance follows that $\operatorname{Diss}_{\mathrm{R}}\left(z_{\varepsilon},[0, t]\right)<+\infty$. Hence, by definition, for any partition $\left\{t_{j}: j=1, \ldots, N\right\}$ of $[0, t]$ we have

$$
\sum_{j=1}^{N} \mathrm{R}\left(\zeta_{\varepsilon}\left(t_{j}\right)-\zeta_{\varepsilon}\left(t_{j-1}\right), \boldsymbol{\pi}_{\varepsilon}\left(t_{j}\right)-\boldsymbol{\pi}_{\varepsilon}\left(t_{j-1}\right)\right)<+\infty
$$

which implies that the map $t \mapsto \zeta_{\varepsilon}(t)$ is non-increasing for the partial ordering " $\leq$ a.e.", hence $\zeta_{\varepsilon}(t)-$ $\zeta_{\varepsilon}(0) \leq 0$ a.e. on $\Gamma_{\mathrm{C}}$. Then, by (5.39) and (5.40) we have

$\mathrm{R}\left(\zeta_{\varepsilon}(t)-\zeta_{\varepsilon}(0), \boldsymbol{\pi}_{\varepsilon}(t)-\boldsymbol{\pi}_{\varepsilon}(0)\right)=\int_{\Gamma_{\mathrm{C}}} \mathrm{a}\left(\zeta_{\varepsilon}(0)-\zeta_{\varepsilon}(t)\right)+\sigma_{\text {yield }}\left|\boldsymbol{\pi}_{\varepsilon}(t)-\boldsymbol{\pi}_{\varepsilon}(0)\right| \mathrm{d} \mathscr{H}^{2} \rightarrow \mathrm{R}(\zeta(t)-\zeta(0), \boldsymbol{\pi}(t)-\boldsymbol{\pi}(0))$

that is $(5.38 \mathrm{~b})$.

Let us prove the strong convergence in $(5.38 \mathrm{e})$. Since, as already remarked, $\mathbf{q}(t)=(\mathrm{u}(t), z(t))$ is an energetic solution to the problem $\left(\mathcal{U} \times \mathcal{Z}, \mathrm{E}_{0}, \mathrm{R}, \mathrm{q}(0)\right)$, then the energy balance

$$
\mathrm{E}_{0}(t, \mathrm{q}(t))+\operatorname{Diss}_{\mathrm{R}}(z,[0, t])=\mathrm{E}_{0}\left(0, \mathbf{q}_{0}\right)+\int_{0}^{t} \partial_{s} \mathrm{E}_{0}(s, \mathbf{q}(s)) \mathrm{d} s
$$


holds true. On the other hand, by the definition of $\overline{\mathrm{E}}$ and since $\mathrm{q}_{\varepsilon}=\left(\mathrm{u}_{\varepsilon}, z_{\varepsilon}\right)$ is an energetic solution to the problem $\left(Q, \mathrm{E}_{\varepsilon}, \mathrm{R}, \mathrm{q}_{\varepsilon}(0)\right)$, for any $\varepsilon>0$ we have also

$$
\mathrm{E}_{\varepsilon}\left(t, \mathrm{q}_{\varepsilon}(t)\right)+\operatorname{Diss}_{\mathrm{R}}\left(z_{\varepsilon},[0, t]\right)=\mathrm{E}_{\varepsilon}\left(0, \mathrm{q}_{0, \varepsilon}\right)+\int_{0}^{t} \partial_{s} \mathrm{E}_{\varepsilon}\left(s, \mathrm{q}_{\varepsilon}(s)\right) \mathrm{d} s .
$$

Substituting

in (5.44) we find

$$
\mathrm{E}_{\varepsilon}\left(t, \mathrm{q}_{\varepsilon}(t)\right)=\overline{\mathrm{E}}_{\varepsilon}\left(t, \mathrm{q}_{\varepsilon}(t)\right)+\int_{\Gamma_{\mathrm{C}}} \frac{\kappa_{0}}{2}\left|\nabla_{\mathrm{S}} \boldsymbol{\pi}_{\varepsilon}\right|^{2} \mathrm{~d} \mathscr{H}^{2}
$$

$$
\int_{\Gamma_{\mathrm{C}}} \frac{\kappa_{0}}{2}\left|\nabla_{\mathrm{S}} \boldsymbol{\pi}_{\varepsilon}\right|^{2} \mathrm{~d} \mathscr{H}^{2}=\mathrm{E}_{\varepsilon}\left(0, \mathrm{q}_{0, \varepsilon}\right)-\overline{\mathrm{E}}_{\varepsilon}\left(t, \mathrm{q}_{\varepsilon}(t)\right)+\operatorname{Diss}_{\mathrm{R}}\left(z_{\varepsilon},[0, t]\right)+\int_{0}^{t} \partial_{s} \mathrm{E}_{\varepsilon}\left(s, \mathrm{q}_{\varepsilon}(s)\right) \mathrm{d} s .
$$

Taking the limit as $\varepsilon \rightarrow 0^{+}$and observing that

- $\mathrm{E}_{\varepsilon}\left(0, \mathrm{q}_{0, \varepsilon}\right) \rightarrow \mathrm{E}_{0}\left(0, \mathrm{q}_{0}\right)$ by assumption,

- $\overline{\mathrm{E}}_{\varepsilon}\left(t, \mathrm{q}_{\varepsilon}(t)\right) \rightarrow \overline{\mathrm{E}}_{0}(t, \mathrm{q}(t))$ by Lemma 5.4 (the fact that $\left(t, \mathrm{q}_{\varepsilon}\right)$ is uniformly stable has been remarked at the beginning of the proof),

- $\operatorname{Diss}_{\mathrm{R}}\left(z_{\varepsilon},[0, t]\right) \rightarrow \operatorname{Diss} R\left(z_{0},[0, t]\right)$ which easily follows by $(5.38 \mathrm{~b})$,

- $\quad \int_{0}^{t} \partial_{s} \mathrm{E}_{\varepsilon}\left(s, \mathrm{q}_{\varepsilon}(s)\right) \mathrm{d} s \rightarrow \int_{0}^{t} \partial_{s} \mathrm{E}_{0}(s, \mathrm{q}(s)) \mathrm{d} s$ by $(5.38 \mathrm{c})$

then we have

$$
\begin{aligned}
\lim _{\varepsilon \rightarrow 0^{+}} \int_{\Gamma_{\mathrm{C}}} \frac{\kappa_{0}}{2}\left|\nabla_{\mathrm{S}} \boldsymbol{\pi}_{\varepsilon}\right|^{2} \mathrm{~d} \mathscr{H}^{2} & =\mathrm{E}_{0}\left(0, \mathrm{q}_{0}(t)\right)-\overline{\mathrm{E}}_{0}(t, \mathrm{q}(t))+\operatorname{Diss}\left(z_{0},[0, t]\right)+\int_{0}^{t} \partial_{s} \mathrm{E}_{0}(s, \mathrm{q}(s)) \mathrm{d} s \\
& =\mathrm{E}_{0}(0, \mathrm{q}(t))-\overline{\mathrm{E}}_{0}(t, \mathbf{q}(t))=\int_{\Gamma_{\mathrm{C}}} \frac{\kappa_{0}}{2}\left|\nabla_{\mathrm{S}} \boldsymbol{\pi}\right|^{2} \mathrm{~d} \mathscr{H}^{2} .
\end{aligned}
$$

Together with the weak convergence, already proven, this implies the strong convergence stated in (5.38e).

Finally, (5.38a) follows by the convergence $\overline{\mathrm{E}}_{\varepsilon}\left(t, \mathrm{q}_{\varepsilon}(t)\right) \rightarrow \overline{\mathrm{E}}_{0}(t, \mathrm{q}(t))$, the definition of $\overline{\mathrm{E}}_{\varepsilon}$ and the strong convergence of $\boldsymbol{\pi}_{\varepsilon}$ to $\boldsymbol{\pi}$.

Arguing as in Section 4 we can provide a purely 2D formulation of the limit problem on $\Gamma_{\mathrm{C}}$ in which the stored energy is

$$
\widehat{\mathrm{E}}_{0}(t, \rho, \xi, z):=\left\{\begin{array}{cc}
\frac{1}{2} \int_{\Gamma_{\mathrm{C}}} \sum_{\ell=1,2}\left(\frac{h_{\ell}}{2} \mathbb{C}^{0} \tilde{e}\left(\rho^{\ell}+\rho_{\mathrm{D}}\right): \tilde{e}\left(\rho^{\ell}+\rho_{\mathrm{D}}\right)+(-1)^{\ell+1} \frac{h_{\ell}^{2}}{8} \mathbb{C}^{0} \tilde{e}\left(\rho^{\ell}+\rho_{\mathrm{D}}\right): \nabla^{2}\left(\xi^{\ell}+\xi_{\mathrm{D}}\right)\right. \\
\left.+\frac{h_{\ell}^{3}}{24} \mathbb{C}^{0} \nabla^{2}\left(\xi^{\ell}+\xi_{\mathrm{D}}\right): \nabla^{2}\left(\xi^{\ell}+\xi_{\mathrm{D}}\right)-\varphi_{3}^{0}\left(\xi^{\ell}+\xi_{\mathrm{D}}\right)-\varphi_{\alpha}^{0}\left(\rho_{\alpha}^{\ell}+\left[\rho_{\mathrm{D}}\right]_{\alpha}\right)+\varphi_{\alpha}^{1} \frac{\partial}{\partial x_{\alpha}}\left(\xi^{\ell}+\xi_{\mathrm{D}}\right)\right) \\
+\zeta 1_{0}\left(q_{\tau}\right) \kappa_{\tau}\left|\rho^{1}-\rho^{2}-\pi\right|^{2}+\zeta 1_{0}\left(q_{\nu}\right) \kappa_{\nu}\left|\xi^{1}-\xi^{2}\right|^{2} \mathrm{~d} \mathscr{H}^{2} & \text { if }(\rho, \xi, \zeta) \in \widehat{\mathrm{A}}_{\mathrm{KL}}^{\mathrm{ad}}, \\
+\infty & \text { else, }
\end{array}\right.
$$

where

$$
\begin{aligned}
\widehat{\mathrm{A}}_{\mathrm{KL}}^{\mathrm{ad}}:=\{ & (\rho, \xi, \zeta) \in W^{1,2}\left(\Gamma_{\mathrm{C}} ; \mathbb{R}^{2}\right)^{2} \times W^{2,2}\left(\Gamma_{\mathrm{C}}\right)^{2} \times L^{\infty}\left(\Gamma_{\mathrm{C}}\right): 0 \leq \zeta \leq 1, \xi^{1} \geq \xi^{2} \text { a.e. on } \Gamma_{\mathrm{C}}, \\
& z\left|\rho^{1}-\rho^{2}\right|=0 \text { if } q_{\tau}<0, z\left(\xi^{1}-\xi^{2}\right)=0 \text { if } q_{\nu}<0, \\
& \left.\left.\rho^{\ell}\right|_{\gamma_{\mathrm{D} \ell}}=0,\left.\quad \xi^{\ell}\right|_{\gamma_{\mathrm{D} \ell}}=\left.\nabla \xi^{\ell}\right|_{\gamma_{\mathrm{D} \ell}} \cdot \nu=0 \text { a.e. on } \gamma_{\mathrm{D} \ell}, \quad \ell=1,2\right\},
\end{aligned}
$$

and the dissipation $\mathrm{R}$ is given in $(5.1 \mathrm{~b})$.

Acknowledgments: The authors thank one of the anonymous referees of [21] for suggesting us to study the sandwich-like plate delamination, and another anonymous referee and the handling editor of this manuscript for many suggestions that improved the present paper. The partial support of FP7-IDEAS-ERC-StG Grant \# 200497 BioSMA and of the grants 201/09/0917, 201/10/0357, and 201/12/067 (GA ČR) is very acknowledged, too. 


\section{References}

[1] E. Acerbi, G. Buttazzo, D. Percivale: A variational definition of the strain energy for an elastic string. J. Elasticity 25 (1991), 137-148.

[2] A.R. Akisanya, N.A. Fleck: Interfcial cracking from the free-edge of a long bi-material strip, Int. J. Solids Strutures 34 (1997), 1645-1665.

[3] G. Anzellotti, S. Baldo, D. Percivale: Dimension reduction in variational problems, asymptotic development in $\Gamma$-convergence and thin structures in elasticity. Asymptotic Anal. 9 (1994), 61-100 (1994).

[4] J.-F. Babadjian, G. Francfort, M.G. Mora: Quasistatic evolution in non-associative plasticity - the cap model. SIAM J. Math. Anal. 44 (2012), 245-292.

[5] M.A. Biot: Thermoelasticity and Irreversible Thermodynamics. J. Appl. Phys. 27(1956), 240-253.

[6] M.A. Biot: Mechanics of Incremental Deformations. Wiley, New York, 1965.

[7] F. Bourquin, P.G. Ciarlet, G. Geymonat, A. Raoult: $\Gamma$-convergence et analyse asymptotique des plaques minces, C.R. Acad. Sci. Paris Sér. I Math. 315 (1992), 1017-1024.

[8] V. Bucur (editor): Delamination in Wood, Wood Products and Wood-Based Composites, Springer, 2011.

[9] P.P. Camanho, C.G. Dávila, D.R. Ambur: Numerical simulation of delamination growth in composite materials, NASA Langley Research Center, 2001.

[10] F. Cagnetti: A vanishing viscosity approach to fracture growth in a cohesive zone model with prescribed crack path. Math. Models Meth. Appl. Sci. 18 (2008), 1027-1071.

[11] P.G. Ciarlet: Mathematical Elasticity, Vol.II: Theory of Plates. North-Holland, Amsterdam, 1997.

[12] P.G. Ciarlet, P. Destuynder: A justification of the two-dimensional linear plate model. J. Mec. Paris 18, (1979) 315-344.

[13] G. Dal Maso, A. DeSimone, M.G. Mora, M. Morini: A vanishing viscosity approach to quasistatic evolution in plasticity with softening. Arch. Ration. Mech. Anal. 189 (2008), 469-544.

[14] G. Dal Maso, A. DeSimone, F. Solombrino: Quasistatic evolution for Cam-Clay plasticity: a weak formulation via viscoplastic regularization and time parametrization, Calc. Var. Partial Differential Equations 40 (2011), $125-181$.

[15] J.P. Dempsey, G.B. Sinclair: On the singular behavior at the vertex of a bi-material wedge. J. Elasticity 11 (1981), 317-327.

[16] M. Efendiev, A. Mielke: On the rate-independent limit of systems with dry friction and small viscosity. $J$. Convex Analysis, 13 (2006), 151-167.

[17] G.A. Francfort, J.-J. Marigo: Stable damage evolution in a brittle continuous medium. Eur. J. Mech., A 12 (1993), 149-189.

[18] G.A. Francfort, J.-J. Marigo: Revisiting brittle fracture as an energy minimization problem. J. Mech. Phys. Solids 46 (1998), 1319-1342.

[19] G. Francfort, A. Mielke: Existence results for a class of rate-independent material models with nonconvex elastic energies. J. reine angew. Math. 595 (2006), 55-91.

[20] L. Freddi, R. Paroni, C. Zanini: Dimension reduction of a crack evolution problem in a linearly elastic plate. Asymptot. Anal. 70 (2010), 101-123.

[21] L. Freddi, R. Paroni, T. Roubíček, C. Zanini: Quasistatic delamination models for Kirchhoff-Love plates. Zeitschift für angew. Math. u. Mech. (ZAMM) 91 (2011), 845-865.

[22] M. Frémond: Dissipation dans l'adhérence des solides. C. R. Acad. Sci. Paris Sér. II Méc. Phys. Chim. Sci. Univers Sci. Terre 300 1985, 709-714.

[23] M. Frémond: Adhérence des solides. J. Mécanique Th. Appl. 6 (1987), 383-407.

[24] M. Frémond: Contact with adhesion. Topics in nonsmooth mechanics, Birkhäuser, Basel, 1988, $157-185$.

[25] G. Geymonat, F. Krasucki, S. Lenci: Mathematical analysis of a bonded joint with a soft thin adhesive. Math. Mech. Solids 4 (1999), 201225.

[26] A.A. Griffith: The phenomena of rupture and flow in solids. Philos. Trans. Royal Soc. London Ser. A. Math. Phys. Eng. Sci. 221 (1921), 163-198.

[27] C. Hwu: Anisotropic elastic plates, Springer, 2010. 
[28] W. S. Johnson (editor): Delamination and Debonding of Materials, ASTM, STP876, Philadelphia, 1985.

[29] A.M. Khludnev: Equilibrium of an elastic plate with an oblique crack. J. Appl. Mech. Tech. Physics 38 (1997), 757-761.

[30] A.M. Khludnev, V.A. Kovtunenko: Analysis of Cracks in Solids. WIT Press, Southampton, Boston, 2000.

[31] D. Knees, A. Mielke, C. Zanini: On the inviscid limit of a model for crack propagation. Math. Models Methods Appl. Sci. 18 (2008), 1529-1569.

[32] D. Knees, A. Schröder: Computational aspects of quasi-static crack propagation. Disc. Cont. Dynamical Systems - Series S (2011), to appear.

[33] D. Knees, C. Zanini, A. Mielke: Crack growth in polyconvex materials. Phys. D 239 (2010), 1470-1484.

[34] J.K. Knowles, E. Sternberg: Large deformations near a tip of an interface-crack between two neo-Hookean sheets. J. Elasticity 13 (1983), 257-293.

[35] M. Kočvara, A. Mielke, T. Roubíček: A rate-independent approach to the delamination problem. Math. Mech. Solids 11 (2006), 423-447.

[36] C.J. Larsen, M. Ortiz, C.L. Richardson: Fracture paths from front kinetics: relaxation and rate independence. Arch. Ration. Mech. Anal. 193 (2009), no. 3, 539-583.

[37] G. Lazzaroni, R. Toader: A model of crack propagation based on viscous approximation. Math. Models Methods Appl. Sci. 21 (2011), 1-29.

[38] H. Le Dret: Problemes Variationnels dans les Multi-domaines. Modélisation des Jonctions et Applications, Masson (1991).

[39] H. Le Dret, A. Raoult: The nonlinear membrane model as variational limit of nonlinear three-dimensional elasticity. J. Math. Pures Appl., IX. Sr. 74 (1995), 549-578.

[40] J. Lemaitre, J.-L. Chaboche: Mechanics of Solid Materials. Cambridge University Press, 1994.

[41] V.I. Levitas: The postulate of realizability: formulation and applications to the post-bifurcation behaviour and phase transitions in elastoplastic materials I, II, Intl. J. Engrg. Sci. 33 (1995), 921-945, 947-971.

[42] K.M. Liechti, Y.S. Chai: Asymmetric shielding in interfacial fracture under in-plane shear. J. Appl. Mech. 59 (1992), 295-304.

[43] M. Liero, A. Mielke: An evolutionary elastoplastic plate model derived via $\Gamma$-convergence. Math. Models Methods Appl. Sci. 21 (2011), 1961-1986.

[44] A. Mainik, A. Mielke: Existence results for energetic models for rate-independent systems. Calc. Var. Partial Differential Equations, 22 (2005), 73-99.

[45] V. Mantič: Discussion on the reference lenght and mode mixity for a bimaterial interface. J. Engr. Mater. Technology 130 (2008) 045501-1-2.

[46] A. Mielke: Evolution of rate-independent systems. Handbook of Differential Equations, Evolutionary Equations, vol. 2, (Eds.: Dafermos, C.M., Feireisl, E.) Elsevier, Amsterdam, 2005, pp.461-559.

[47] A. Mielke: Differential, energetic and metric formulations for rate-independent processes. In: Nonlinear PDEs and Applications (Eds. L.Ambrosio, G.Savaré.) Springer, 2010, pp.87-170.

[48] A. Mielke, R. Rossi, G. Savaré: BV solutions and viscosity approximations of rate-independent systems. (WIAS preprint 1451, Berlin, 2009) ESAIM Control Optim. Calc. Var. (2011), in print. (DOI: $10.1051 / \mathrm{cocv} / 2010054)$

[49] A. Mielke, T. Roubíček: Numerical approaches to rate-independent processes and applications in inelasticity. Math. Model. Numer. Anal. 43 (2009), 399-428.

[50] A. Mielke, T. Roubíček, U. Stefanelli: $\Gamma$-limits and relaxations for rate-independent evolutionary problems. Calc. Var. Partial Differential Equations. 31 (2008), 387-416.

[51] A. Mielke and F. Theil: On rate-independent hysteresis models, Nonlin. Diff. Equations Appl. 11 (2004), 151-189. (Accepted July 2001).

[52] A. Mielke, F. Theil, V.I. Levitas: A variational formulation of rate-independent phase transformations using an extremum principle. Arch. Rat. Mech. Anal. 162 (2002), 137-177.

[53] A. Mielke, S. Zelik: On the vanishing-viscosity limit in parabolic systems with rate-independent dissipation terms. Ann. Sc. Norm. Super. Pisa Cl. Sci. (5) (2012), to appear. 
[54] M. Negri, C. Ortner: Quasi-static crack propagation by Griffith's criterion. Math. Models Methods Appl. Sci. 18 (2008), 1895-1925.

[55] M. Negri: A comparative analysis on variational models for quasi-static brittle crack propagation. Adv. Calc. Var. 3 (2010), 149-212.

[56] C. G. Panagiotopoulos, V. Mantič, T. Roubíček: BEM implementation of energetic solutions for quasistatic delamination problems. In: Advances in Boundary Element Techniques XII (BeTeq 2011) E.L. Albuquerque, M.H. Aliabadi (Eds.), Engineering Conferences Ltd., Eastleigh, 2011, pp. 261-268.

[57] R. Paroni, P. Podio Guidugli: On certain widespread ideas for dimension reduction in structure mechanics. In preparation.

[58] J.R. Rice: Elastic fracture mechanics concepts for interfacial cracks, J. Appl. Mech. (Trans. ASME) 55 (1988), 98-103.

[59] T. Roubíček, M. Kružík, J. Zeman: Delamination and adhesive contact models and their mathematical analysis and numerical treatment. In: Math. Methods and Models in Composites (Chap.9). (V. Mantič, ed.) Imperial College Press, ISBN 978-1-84816-784-1. 2012, in print.

[60] T. Roubíček, V. Mantič, C.G. Panagiotopoulos: Quasistatic mixed-mode delamination model. (Preprint No. 2011-020, Nečas center, Prague) Disc. Cont. Dynam. Syst., Ser. S 6 (2013), in print.

[61] T. Roubíček, L. Scardia, C. Zanini: Quasistatic delamination problem. Cont. Mech. Thermodynam. 21 (2009), 223-235.

[62] M. Sofonea, W. Han, M. Shillor: Analysis and approximation of contact problems with adhesion or damage. Pure and Applied Mathematics (Boca Raton) 276. Chapman \& Hall/CRC, Boca Raton, FL, 2006.

[63] L. Távara, V. Mantič, E. Graciani, F. París: BEM analysis of crack onset and propagation along fiber-matrix interface under transverse tension using a linear elastic-brittle interface model. Eng. Anal. Bound. Elem. 35 (2) (2011), 207222.

[64] V. Tvergaard, J.W. Hutchinson: The influence of plasticity on mixed mode interface toughness. J. Mech. Phys. Solids 41 (1993), 1119-1135. 